\title{
Counteracting the effect of reducing nitrate/nitrite levels on dry fermented sausage aroma by Debaryomyces hansenii inoculation
}

Laura Perea-Sanz, José Javier López-Díez, Carmela Belloch, Mónica Flores*

Instituto de Agroquímica y T ecnología de Alimentos (IATA-CSIC), Avda. Agustín Escardino 7, 46980 Paterna, Valencia, Spain

\begin{abstract}
The reduction of ingoing amounts of nitrate and nitrite in dry fermented sausages was studied together with the impact of Debaryomyces hansenii inoculation on aroma generation. Three different formulations of sausages were manufactured: control $(C)$, reduced in nitrate and nitrite ingoing amounts ( $\mathrm{R}$ ) and reduced $\mathrm{R}$ inoculated with D. hansenii (RY). Changes in physicochemical and microbiological parameters, volatile compounds and aroma were investigated at different drying times. Nitrite/nitrate reduction did not seem to affect microbial growth but affected their metabolic activity. Moreover, nitrite/nitrate reduction decreased lipid oxidation and generation of derived volatile compounds. Yeast inoculation limited lipid oxidation and prevented nitrite oxidation. Sausage aroma profile was positively affected by $D$. hansenii inoculation which contributed to the generation of potent aroma compounds like ethyl ester compounds and 3-methylbutanal. Long drying time impacted sausage aroma profile as well as yeast metabolism. Yeast inoculation counteracted the negative influence of nitrite/nitrate reduction due to its antioxidant capacity, aroma generation and hindered nitrite oxidation.
\end{abstract}

Keywords: Nitrate; Nitrite; D. hansenii; Fermented sausage; Volatile compounds; Aroma 


\section{Introduction}

The use of nitrite and nitrate as curing agents in dry fermented sausages have important technological functions. Nitrite has antioxidant and antimicrobial activities being the most important its activity against the pathogen Clostridium botulinum and control of toxin production. Furthermore, nitrite facilitates the generation and stabilization of the typical colour and enhances cured flavour (Honikel, 2008; Sindelar \& Milkowski, 2011). Nitrate acts as nitrite reservoir in dry cured products with a long ripening time (Sanchez Mainar \& Leroy, 2015). However, the controversy about the adequate amounts of nitrite used as additive in meat products processing is under concern (CODEX, 2019). Nitric oxide, from nitrite, can react with biogenic amines present in meat under heating conditions to form $\mathrm{N}$-nitrosamines, with carcinogenic potential (De Mey, De Maere, Paelinck \& Fraeye, 2015). Therefore, the actual trend is to reduce the use of nitrite and nitrate in cured meat products (EFSA, 2010), although nitrite is considered beneficial for improving the microbial safety of the products. Up to now, several alternatives to nitrite addition have been studied. Among them, plant derivatives are one of the most used, followed by organic acids, bacteria and bacteriocins (Alahakoon, Jayasena, Ramachandra \& Jo, 2015). However, these alternatives do not provide all nitrite functions in dry cured fermented sausages. Nitrate and nitrite reduction below their allowed maximum levels in European legislation $(150 \mathrm{mg} / \mathrm{kg}$ of sodium nitrate and $150 \mathrm{mg} / \mathrm{kg}$ of sodium nitrite, (Regulation (EC) no $1333 / 2008$ ) have safety and sensory consequences. The reduction up to a $50 \%$ of the allowed limits affects growth of pathogenic microorganisms (Hospital, Hierro \& Fernandez, 2014), volatile compounds production (Hospital, Hierro, Stringer \& Fernandez, 2016) and aroma profile (Perea-Sanz, Montero, Belloch \& Flores, 2018). A recent study (Christieans, Picgirard, Parafita, Lebert \& Gregori, 2018) has shown that the use of a mixture of sodium nitrate and sodium nitrite at a concentration of $80 \mathrm{mg} / \mathrm{kg}$, was able to control the growth of Listeria monocytogenes and Salmonella typhimurium. Nevertheless, the importance of nitrite presence at the beginning of the process to control these pathogens was remarked due to the slow conversion of nitrate into nitrite (Christieans, et al., 2018).

The consequences of the use of reduced nitrate and nitrite mixtures on aroma profile of dry cured meat products have been scarcely studied. The inoculation of yeasts such as Debaryomyces (D.) hansenii with potential to generate desirable aromas in dry fermented sausages is a suitable alternative. This yeast has been shown to produce esters and sulfur compounds as cured aroma contributors to the aroma profile of dry fermented sausages (CanoGarcia, Belloch \& Flores, 2014; Perea-Sanz, Peris, Belloch \& Flores, 2019a). The production of aroma compounds by $D$. hansenii strains is influenced by the concentration of curing agents (Flores, Moncunill, Montero, López-Díez \& Belloch, 2017), as well as by the availability of specific amino acids used as volatile precursors (Perea-Sanz et al., 2019a). A recent study 
regarding the production of volatile sulphur compounds that contribute to meat aroma notes by D. hansenii strains revealed that one yeast strain may have an impact on dry fermented sausage aroma due to its ability to express a gene directly related to sulfur compound production (PereaSanz et al., 2019a). Consequently, the aim of the present study is to evaluate the effect of the inoculation of a $D$. hansenii strain on dry fermented sausages manufactured with reduced ingoing amounts of nitrite and nitrate and its effect on aroma production during the ripening time.

\section{Materials and methods}

\subsection{Dry fermented sausages manufacture}

Three different formulations of dry fermented sausages were manufactured. Control (C), containing sodium nitrite at $150 \mathrm{ppm}$ and potassium nitrate at $178 \mathrm{ppm}$, and two formulations with a $47 \%$ reduction of both nitrite and nitrate (80 and $95 \mathrm{mg} / \mathrm{kg}$, respectively) ingoing amounts (R) as proposed by Christieans et al. (2018), but one of them inoculated with $D$. hansenii strain L1 (RY). Three replicates of the experiment were performed. Pork's lean meat and belly fat (boneless and skinless) were purchased from a local producer (Cárnicas La Cope, Torrente, Spain). Lean (50\% lean pork meat) and fat (50\% pork belly) were ground through a 10 $\mathrm{mm}$ diameter minced plate and mixed with the following ingredients: $20 \mathrm{~g} / \mathrm{kg}$ lactose, $20 \mathrm{~g} / \mathrm{kg}$ dextrin, $6 \mathrm{~g} / \mathrm{kg}$ glucose, $20.25 \mathrm{~g} / \mathrm{kg}$ sodium chloride $(\mathrm{NaCl}), 6.75 \mathrm{~g} / \mathrm{kg}$ potassium chloride $(\mathrm{KCl})$, $0.5 \mathrm{~g} / \mathrm{kg}$ sodium ascorbate and $1.5 \mathrm{~g} / \mathrm{kg}$ black pepper. Commercial starter culture TRADI-302 (Danisco, Cultor, Madrid, Spain) containing Lactobacillus sakei, Staphylococcus xylosus and Staphylococcus carnosus was added to the meat $(0.125 \mathrm{~g} / \mathrm{kg})$. In the three replications, each formulation was supplemented with the different ingoing amounts of nitrate/nitrite mixtures. Then, D. hansenii L1 was inoculated using $10 \mathrm{ml}$ saline suspension containing approximately 6 $\mathrm{x} 10^{9}$ cells $/ \mathrm{g}$. This yeast suspension was added to $6 \mathrm{~kg}$ of meat batter to reach a final concentration of $10^{6}$ cells $/ \mathrm{g}$. To homogenize all ingredients into the meat batter, a mixerkneading machine provided with rotating blades was used. Formulations were kept at $3-5^{\circ} \mathrm{C}$ for $24 \mathrm{~h}$ to rest after yeast inoculation and then, stuffed into $95 \mathrm{~mm}$ diameter collagen casings (Fibran, S.A., Girona, Spain), being the final weight of each sausage approximately $500 \mathrm{~g}$. All sausages were submitted to a slow ripening process and dried at $10{ }^{\circ} \mathrm{C}$ and $75-90 \%$ relative humidity (RH). In order to control the ripening process, temperature and $\mathrm{RH}$ of the ripening chambers were continuously recorded. A total of 9 batches $(3 \times 3)$ were produced. One sausage from each formulation was weighed regularly to control weight losses. Also, one sausage per formulation was used to control the $\mathrm{pH}$ by introducing a $\mathrm{pH}$ meter HI 99163 (Hanna Instruments Inc., Hoonsocket, USA) into the sausage centre as described by Olivares, Navarro, Salvador \& Flores (2010). Sausages from the different formulation and replicates were sampled for analyses at 0,60 and $83 \mathrm{~d}$ of process. At $0 \mathrm{~d}$, approximately $200 \mathrm{~g}$ of the minced meat 
mixture were collected for analysis. After $60 \mathrm{~d}$ of ripening (44-45\% weight loss), three sausages from each formulation and replicate ( $3 \times 3$ sausages) were randomly chosen for analyses. The remaining sausages were ripened for 23 additional days (50\% weight loss), and afterwards they were collected as above. Slices were taken for microbial analyses ( $25 \mathrm{~g})$ and other slices for volatile analysis were vacuum packed and stored at $-80^{\circ} \mathrm{C}$. Sausage colour and physicochemical analysis (moisture, water activity aw, $\mathrm{pH}$, TBARS, protein and residual nitrite and nitrate content) were measured on minced sausage.

\subsection{Physicochemical parameters measurement}

Measurements of $\mathrm{pH}, \mathrm{a}_{\mathrm{w}}$, weight loss, colour (CIE L*, $\left.\mathrm{a}^{*}, \mathrm{~b}^{*}\right)$, moisture, and protein was done as described in Perea-Sanz et al. (2018). Lipid autoxidation was measured by the thiobarbituric acid reactive substances (TBARS) method and results expressed as $\mu \mathrm{g}$ malonaldehyde (MDA)/g in dry matter (Perea-Sanz et al., 2018). Residual nitrate and nitrite contents were extracted by Carrez precipitation and determined after zinc reduction and Griess reaction as reported in Merino (2009). Measurements were expressed as $\mathrm{mg} / \mathrm{kg}$ of sodium nitrite and potassium nitrate in dry matter.

\subsection{Microbiological analysis}

Microbial counts of lactic acid bacteria (LAB), gram positive coagulase negative cocci (GCN), yeasts and moulds and enterobacteria were carried out as described in Perea-Sanz et al. (2018).

\subsection{D. hansenii L1 monitoring by M13 RAPD PCR}

Fifteen yeast colonies were isolated (Perea-Sanz et al., 2018) from each sausage batch (C, R and RY) at the three ripening times $(0,60$ and $83 \mathrm{~d})$. Colonies were tested for purity and freshly isolated colonies selected and cultured overnight at $25{ }^{\circ} \mathrm{C}$ in $5 \mathrm{ml}$ of GPY (glucose $2 \%$, peptone $0.5 \%$ and yeast extract $0.5 \%$ ). DNA extraction and M13 minisatelite PCR amplification were carried out as described in Cano-García, Flores \& Belloch. (2013). PCR products were separated by electrophoresis on $2 \%$ agarose gel in $1 \mathrm{X}$ TAE buffer at $90 \mathrm{~V}$ for $3 \mathrm{~h}$, stained with Red safe nucleic acid staining solution 20000x (Intron biotechnology, Korea) and visualized under UV light. DNA fragment sizes were determined using 100 bp DNA ladder (Invitrogen, USA).

\subsection{Volatile compound analysis}

Analysis of volatile compounds was carried out by headspace (HS) solid-phase microextraction (SPME) with an $85 \mu \mathrm{m}$ carboxen/polydimethylsiloxane (CAR/PDMS) fibre (Supelco, Bellefonte, USA) using a gas chromatograph (Agilent HP 7890 series II, Hewlett-Packard, Palo Alto, USA) with a quadrupole mass detector (HP 5975C, Hewlett-Packard, Palo Alto, USA) and equipped with an autosampler (MPS2 multipurpose sampler (Gerstel, Germany). In summary, 5 $\mathrm{g}$ of sausage supplemented with $0.75 \mathrm{mg}$ BHT to avoid oxidation, were placed into a headspace 
vial and incubated at $37^{\circ} \mathrm{C}$ for $30 \mathrm{~min}$. The extracted volatile compounds were adsorbed in the fibre for $90 \mathrm{~min}$ at $37^{\circ} \mathrm{C}$ and desorbed in the injection port of the GC-MS for $5 \mathrm{~min}$ at $240{ }^{\circ} \mathrm{C}$ in splitless mode. The volatile compounds were separated using a DB-624 capillary column (30 m, $0.25 \mathrm{~mm}$ i.d., film thickness $1.4 \mu \mathrm{m}$, (J\&W Scientific, Agilent Technologies, USA)) using the conditions described by Corral, Salvador, Belloch \& Flores. (2015). The MS interface temperature was set to $240{ }^{\circ} \mathrm{C}$. The compounds were identified in full scan mode and by comparison with mass spectra from the library database (Nist'05), with linear retention indices (Van Den Dool \& Kratz, 1963) and with authentic standards. The quantitation was done in SCAN mode using either total or extracted ion current (TIC or EIC) on an arbitrary scale and expressed as abundance units (AU) $\times 10^{5}$.

\subsection{Olfactometric analysis}

The analysis of aroma compounds was carried out in a gas chromatograph (Agilent 6890, USA) equipped with a FID detector and a sniffing port (ODP3, Gerstel, Mülheim an der Ruhr, Germany) as described in Corral et al. (2015). Each assessment was carried out on $5 \mathrm{~g}$ of sausage using the detection frequency method (Pollien et al., 1997). The olfactometry analysis was done in sausages ripened for $60 \mathrm{~d}$. Five trained panellists evaluated the odours from the GC-effluent. A total of 12 assessments were carried out. Aroma compounds were identified by comparison with mass spectra, using linear retention indices of authentic standards injected in GC-MS and GC-O and by the coincidence of the assessor's descriptors with those in the Fenaroli's handbook of flavour ingredients (Burdok, 2002).

\subsection{Statistical analysis}

Data were analysed using the Generalized Linear Model (GLM) procedure of statistical software (XLSTAT 2011, v5.01, Addinsoft, Barcelona, Spain). Data were analysed using the linear mixed model including sausage formulation and ripening time as fixed effects, and replicates as random effect. The interaction between fixed effects was tested and evaluated as not significant, therefore it was excluded from the model. When a significant effect of the treatment group was detected ( $\mathrm{P}<0.05$ ), least squares means (LSM) were compared using Tukey test. Principal component analysis (PCA) was plotted to evaluate the relationships among sausage formulations and parameters (physicochemical, microbiological and aroma compounds) measured at each sampling time.

\section{Results}

\subsection{Physicochemical characteristics of the sausages}

Measurements of physicochemical parameters from samples of the three formulations at the three sampling times are shown in Table 1 . At the initial time $(0 \mathrm{~d})$, the three formulations were very similar regarding all parameters except nitrite and nitrate concentration. Residual nitrite 
differences between $\mathrm{C}$ and reduced $\mathrm{R}$ and $\mathrm{RY}$ formulations was $45-52 \%$, whereas residual nitrate differences were about $31-33 \%$. After $60 \mathrm{~d}$ of ripening, no residual nitrite levels were detected in any formulation, while residual nitrate levels in R and RY respect to C were $40 \%$ and $46 \%$, respectively. These differences in residual nitrate concentrations were maintained after $83 \mathrm{~d}$ of ripening. During the ripening time, residual nitrate decreased around $17 \%, 36 \%$ and $48 \%$ within C, R and RY sausages, respectively. Colour parameter a* increased after $60 \mathrm{~d}$ of ripening. Lipid oxidation (TBARS) evolution was different among the formulations. $\mathrm{C}$ and $\mathrm{R}$ showed the same trend, an initial increase at $60 \mathrm{~d}$ and a slow decline after $83 \mathrm{~d}$, while RY sausages did not show lipid oxidation maintaining low TBARS values during the ripening time. In addition, the highest $\mathrm{pH}$ value was reached at $60 \mathrm{~d}$ in $\mathrm{RY}$ sausages. These differences between RY and the other two formulations could be observed also in water activity and moisture at $83 \mathrm{~d}$ of ripening time.

\subsection{Microbiological counts}

Results on microbial counts are shown in Fig. 1. Enterobacteriaceae were not found in any sausage batch at any sampling time (data not shown). LAB counts (Fig. 1A) increased from $0 \mathrm{~d}$ to $60 \mathrm{~d}$ and decreased slightly at $83 \mathrm{~d}$. Significant differences between LAB counts were found at $0 \mathrm{~d}$ in $\mathrm{RY}$ respect to $\mathrm{C}$ and $\mathrm{R}$ batches, but at 60 and $83 \mathrm{~d}$ LAB counts were indistinguishable between batches. GCN counts (Fig. 1B) decreased constantly from $0 \mathrm{~d}$ and no significant differences were found among sausages at any ripening time. Yeast counts (Fig. 1C) were lower than $10 \mathrm{cfu} / \mathrm{g}$ in $\mathrm{C}$ ( 2 colonies) and R (5 colonies) sausages at $0 \mathrm{~d}$, whereas the RY showed a yeast count in accordance with $D$. hansenii L1 inoculation. Yeast counts increased from $0 \mathrm{~d}$ to $60 \mathrm{~d}$ in all sausages and decreased slightly from 60 to $83 \mathrm{~d}$, however counts in RY were higher than $\mathrm{C}$ and $\mathrm{R}$.

\subsection{D. hansenii monitoring by M13 RAPD-PCR along sausage ripening}

The implantation of the inoculated D. hansenii strain on RY sausages, as well as its presence in C and R sausages, was followed by comparison of M13 minisatellite PCR patterns of yeast isolates with the inoculated D. hansenii L1 strain (Fig. 2). M13 patterns of yeast isolates from RY sausages at any ripening time were identical to the pattern of the inoculated $D$. hansenii L1 strain. On the other hand, several yeast isolates from $\mathrm{C}$ sausages (Figure 2A) and R sausages (Figure 2B) showed M13 patterns different from the pattern of D. hansenii L1 strain. In C sausages, only one isolate at $60 \mathrm{~d}$ and in $\mathrm{R}$ batch 5 isolates at $60 \mathrm{~d}$ and 6 isolates at $83 \mathrm{~d}$ were found with identical pattern of the inoculated D. hansenii L1 strain. 

Table 1. Effect of nitrite/nitrate reduction and $D$. hansenii inoculation on physicochemical parameters of dry fermented sausages.

\begin{tabular}{|c|c|c|c|c|c|c|c|c|c|c|c|c|c|c|c|c|c|c|c|c|}
\hline & \multicolumn{6}{|c|}{$\mathrm{C}^{1}$} & \multicolumn{5}{|c|}{$\mathbf{R}$} & \multicolumn{6}{|c|}{ RY } & \multirow[b]{2}{*}{ RMSE $^{2}$} & \multirow[b]{2}{*}{$\boldsymbol{P}_{\mathrm{t}}^{3}$} & \multirow[b]{2}{*}{$P_{\mathrm{f}}$} \\
\hline & Od & & $60 d$ & & 83d & & $\mathbf{0 d}$ & & $60 d$ & & 83d & Od & & 60d & & 83d & & & & \\
\hline $\mathbf{p H}$ & 5.79 & $\mathrm{a}$ & 4.85 & $\mathrm{~d}$ & 4.94 & $\mathrm{bc}$ & 5.79 & $\mathrm{a}$ & 4.86 & $\mathrm{~d}$ & $4.94 \mathrm{bc}$ & 5.80 & $\mathrm{a}$ & 4.99 & $\mathrm{~b}$ & 4.92 & $\mathrm{c}$ & 0.05 & $* * *$ & $* *$ \\
\hline aw & 0.973 & $\mathrm{a}$ & 0.894 & $\mathrm{~b}$ & 0.809 & $d$ & 0.973 & $\mathrm{a}$ & 0.897 & $\mathrm{~b}$ & $0.807 \mathrm{~d}$ & 0.969 & $\mathrm{a}$ & 0.899 & $\mathrm{~b}$ & 0.837 & $\mathrm{c}$ & 0.006 & $* * *$ & $* * *$ \\
\hline Moisture \% & 64.15 & $\mathrm{a}$ & 42.44 & $\mathrm{~b}$ & 29.69 & $d$ & 64.19 & $\mathrm{a}$ & 42.28 & $\mathrm{~b}$ & $29.77 \mathrm{~d}$ & 63.48 & $\mathrm{a}$ & 41.92 & $\mathrm{~b}$ & 32.58 & $\mathrm{c}$ & 1.18 & $* * *$ & ns \\
\hline Protein $(\% \mathrm{dm})$ & 49.42 & $\mathrm{~d}$ & 52.89 & $\mathrm{~cd}$ & 54.14 & bc & 50.71 & $\mathrm{~cd}$ & 58.08 & $\mathrm{a}$ & $53.23 \mathrm{bcd}$ & 49.97 & d & 56.22 & $a b$ & 53.25 & bcd & 2.81 & $* * *$ & ns \\
\hline $\mathbf{L}^{*}$ & 59.81 & $\mathrm{a}$ & 52.85 & $\mathrm{~b}$ & 44.76 & $\mathrm{~cd}$ & 59.91 & $\mathrm{a}$ & 52.21 & $\mathrm{~b}$ & $44.34 \mathrm{~d}$ & 60.26 & $\mathrm{a}$ & 53.45 & $\mathrm{~b}$ & 46.64 & $\mathrm{c}$ & 1.71 & $* * *$ & ns \\
\hline$a^{*}$ & 13.79 & $\mathrm{c}$ & 16.81 & $\mathrm{a}$ & 14.40 & bc & 14.49 & $\mathrm{bc}$ & 16.76 & $\mathrm{a}$ & $14.24 \mathrm{bc}$ & 12.64 & d & 17.04 & $\mathrm{a}$ & 14.78 & $\mathrm{~b}$ & 0.51 & $* * *$ & ns \\
\hline TBARS $^{4}$ & 0.59 & $\mathrm{~d}$ & 3.62 & $\mathrm{a}$ & 2.21 & $\mathrm{~b}$ & 0.48 & $\mathrm{~d}$ & 2.23 & $\mathrm{~b}$ & $1.27 \mathrm{c}$ & 0.47 & $\mathrm{~d}$ & 0.63 & $\mathrm{~d}$ & 0.47 & $\mathrm{~d}$ & 0.32 & $* * *$ & $* * *$ \\
\hline $\mathrm{NaNO}_{2} \mathrm{mg} / \mathrm{kg} \mathrm{dm}$ & 217.40 & $\mathrm{a}$ & 0.00 & $\mathrm{c}$ & 0.00 & $\mathrm{c}$ & 102.32 & $\mathrm{~b}$ & 0.00 & $\mathrm{c}$ & $0.00 \mathrm{c}$ & 119.10 & $\mathrm{~b}$ & 0.00 & $\mathrm{c}$ & 0.00 & $\mathrm{c}$ & 11.20 & $* * *$ & $* * *$ \\
\hline $\mathrm{KNO}_{3} \mathrm{mg} / \mathrm{kg} \mathrm{dm}$ & 533.05 & $\mathrm{a}$ & 396.77 & $\mathrm{bc}$ & 442.44 & $\mathrm{~b}$ & 363.81 & $\mathrm{c}$ & 235.32 & $\mathrm{~d}$ & $233.20 \mathrm{~d}$ & 364.66 & c & 212.67 & d & 188.39 & d & 42.37 & $* * *$ & $* * *$ \\
\hline
\end{tabular}

${ }^{1} \mathrm{C}$ : control batch; R: 47\% reduction in nitrite and nitrate; RY: 47\% reduction in nitrite and nitrate and $D$. hansenii inoculated. ${ }^{2} \mathrm{RMSE}$ : root mean square error. ${ }^{3} P_{\mathrm{t}}: P$ value of time effect, $P_{\mathrm{f}}: P$ value of formulation effect. Different letters in the same row of each group indicate significant differences at $* * * \mathrm{P}<0.001$, $* * \mathrm{P}<0.01, * \mathrm{P}<0.05$. ns: $\mathrm{P}>0.05$. ${ }^{4}$ TBARS expressed as $\mu \mathrm{g}$ malonaldehyde $/ \mathrm{g} \mathrm{dm}$. 


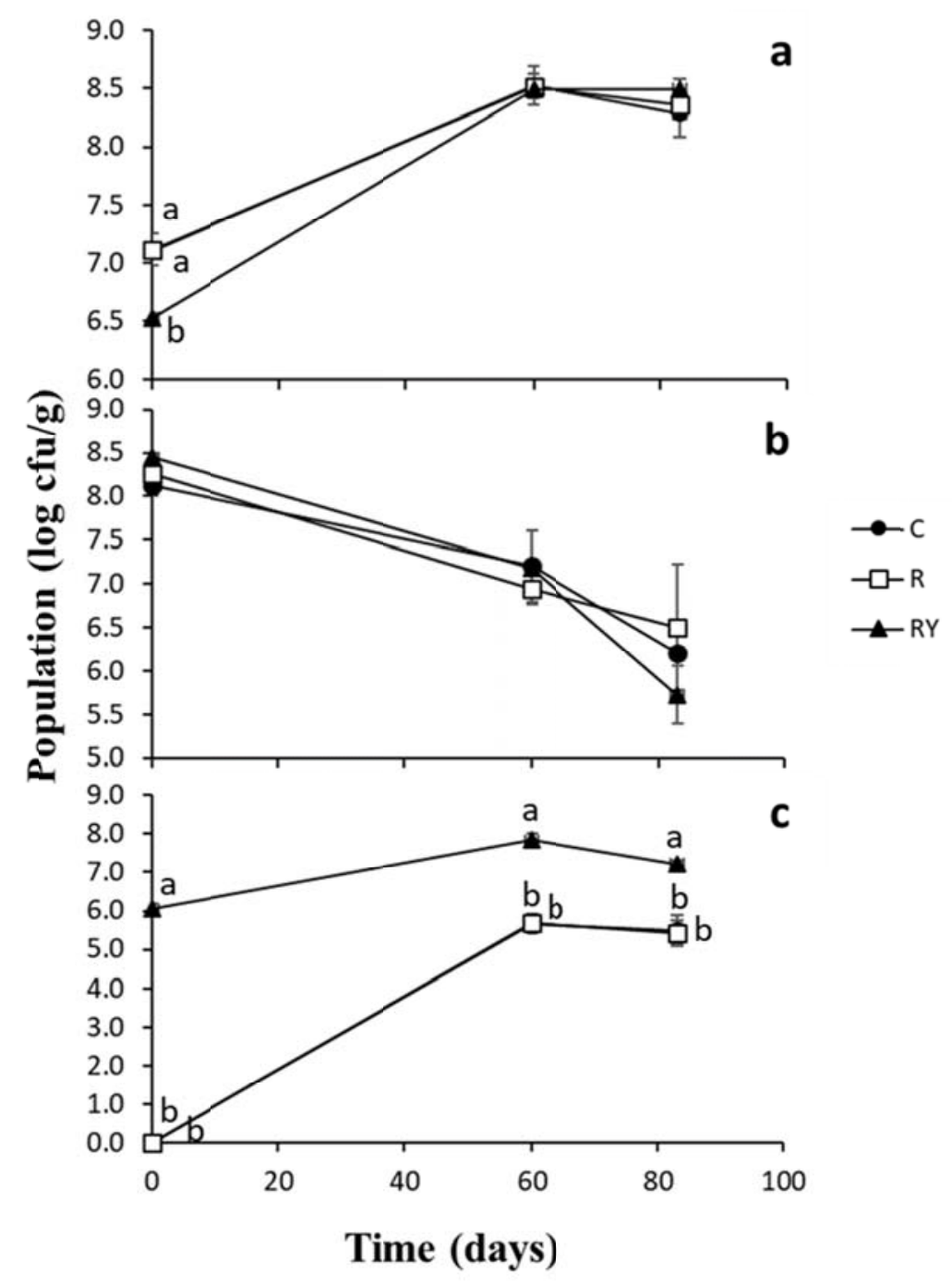

Figure 1. Changes in microbial populations during the ripening of dry fermented sausages in Control (•), R ( $\square$ ) and RY ( $\mathbf{\Delta})$ formulations. Lactic acid bacteria (LAB) (a), Gram positive coagulase negative cocci $(\mathrm{GCN})(\mathrm{b})$ and yeasts and moulds (c). Different letters in the same ripening time indicate mean differences at $* * * \mathrm{P}<0.001$ among sausages. 
A

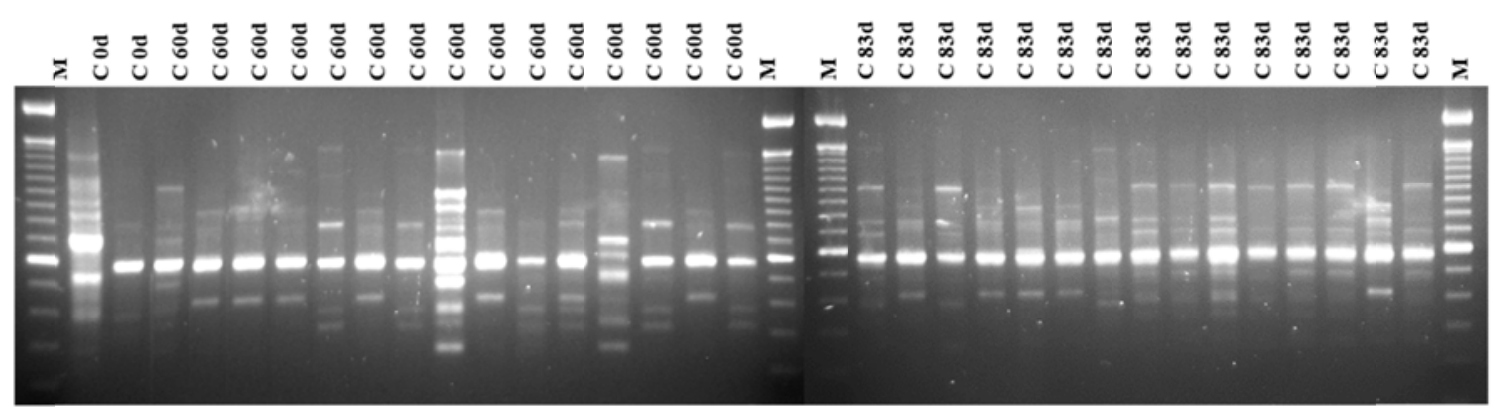

B

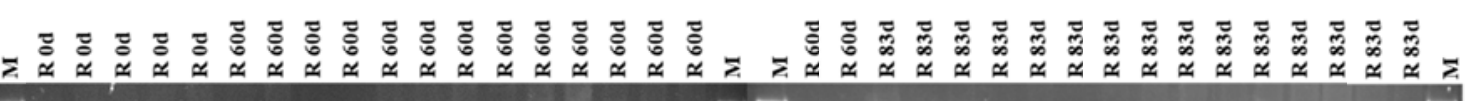

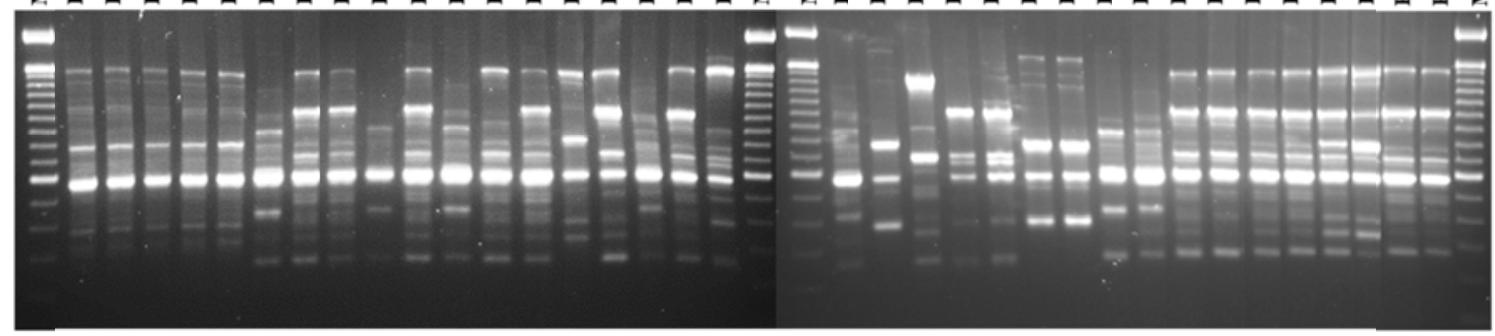

C

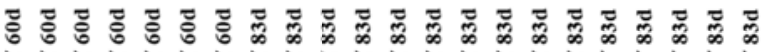

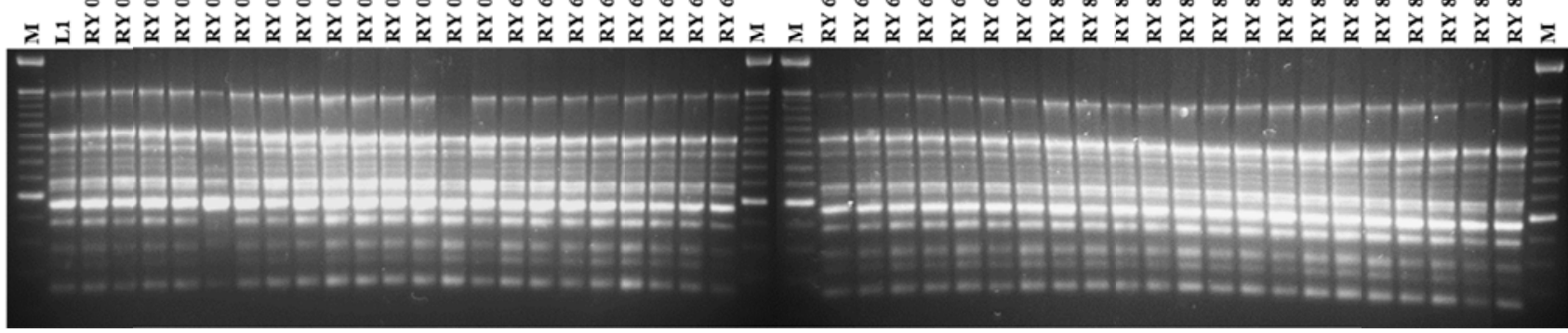

Figure 2. Electrophoretic patterns of minisatellite M13 PCR amplification of yeast isolates at 0,60 and $83 \mathrm{~d}$ of ripening in C (A), R (B) and RY (C) formulations. The arrow indicates bands of isolated yeast in $\mathrm{C}$ and $\mathrm{R}$ sausages matching the pattern of the inoculated strain L1. Lane $\mathrm{M}$ is the $100 \mathrm{bp}$ ladder.
Lane
L1
in
panel
C represents
the pattern
of
the
inoculated
D. hansenii
strain. 


\subsection{Volatile compounds generation}

Eighty volatile compounds were identified and quantified in the HS of dry fermented sausages using a CAR/PDMS fibre (Table 2). The volatile compounds were classified by their most probable origin. Within the bacterial metabolism origin, four subgroups were done: amino acid degradation (17), carbohydrate fermentation (8), esterase activity (10) and $\beta$-oxidation (1)), from the chemical reaction origin two subgroups: lipid oxidation reaction (22) and thiamine degradation (2)); and finally, from spices (17) and from unknown origin (3). Among the 80 volatiles identified, 61 compounds were confirmed while 11 were tentatively identified.

The RY sausages showed the highest concentration of volatile compounds from amino acid degradation at both drying times. The most abundant compounds were 3-methyl-1-butanol, 2methyl propanoic acid and 2-methyl and 3-methyl butanoic acids, whereas dimethyl disulphide, toluene, 3-(methylthio)propanal, benzeneacetaldehyde, 2,6-dimethylpyrazine and phenol were the less abundant. On the other hand, methanethiol and 3-methylbutanal were the most abundant compounds found in $\mathrm{C}$ and $\mathrm{R}$ sausages. Comparison between $\mathrm{C}$ and $\mathrm{R}$ sausages revealed that nitrite and nitrate reduction produce significant differences only in two compounds; 3(methylthio)propanal and benzeneacetaldehyde which were more abundant in $\mathrm{C}$ sausages. During the ripening period between 60 and $83 \mathrm{~d}$, the total volatile compounds derived from amino acid degradation diminished in RY sausages. This decrease was significant in methanethiol, 2-methylpropanal, 3-methylbutanal, 2-methylbutanal, and 3-methyl-1-butanol concentrations. Only benzeneacetaldehyde increased after the $60 \mathrm{~d}$ of ripening in RY sausages. Three volatile compounds derived from amino acid degradation were identified and only traces were detected of 2-acetyl-2-thiazoline, 2-acetyl-1-pyrroline and dimethyl trisulfide.

The sum of carbohydrate fermentation volatiles was significantly higher in $\mathrm{C}$ and $\mathrm{R}$ sausages than in RY sausages at both drying times. The most abundant compounds in $\mathrm{C}$ and $\mathrm{R}$ sausages respect to RY sausages were 2,3-butanedione, 2-butanone, acetic acid and 3-hydroxy-2butanone. On the contrary, acetaldehyde and ethyl alcohol were present in significant high proportions in RY sausages. During the ripening time between 60 and $83 \mathrm{~d}$, the total abundance of volatile compounds derived from carbohydrate fermentation increased exclusively in $\mathrm{C}$ sausages due to the increase in acetic acid.

Ethyl acetate was the most abundant volatile compound derived from esterase activity in all sausage types, followed by ethyl-3-methylbutanoate in RY sausages. The total abundance of volatile compounds derived from esterase activity was significantly higher in RY sausages than in $\mathrm{C}$ and $\mathrm{R}$ sausages at both drying times. This difference was observed in all ester compounds except for ethyl pentanoate and ethyl hexanoate. In addition, 3-methylbutyl acetate and ethyl-2methylpropanoate were exclusively present in RY sausages. Nitrate and nitrite reduction in $\mathrm{R}$ 
produced a decrease in two ester compounds, ethyl hexanoate and ethyl 2-hydroxypropanoate at $60 \mathrm{~d}$ although the additional drying time increased its abundance.

Among volatile compounds derived from chemical origin only those derived from lipid oxidation were analysed being the most abundant hexanal in all sausages. These compounds were the lowest in RY sausages at both ripening times. This was due to a significant decrease of most volatiles except for 1-propanol. The last step of ripening from 60 to $83 \mathrm{~d}$, caused a decrease in the total abundance of volatile compounds derived from lipid oxidation although pentanoic and octanoic acids increased during this period.

Only two volatile compounds from thiamine degradation (2-methyl-3-furanthiol and methyl-2methyl-3-furyldisulfide) were identified as traces. Finally, volatile compounds derived from spices were abundant in all sausage formulations but most of them were not affected by either formulation or drying time. 
Table 2. Effect of nitrite/nitrate reduction and $D$. hansenii inoculation on volatile compounds generated in dry fermented sausages (expressed as abundance (AU) of total ion current (TIC) or area of the target ion shown in parenthesis as $\mathrm{AU} \times 10^{5}$ ).

\begin{tabular}{|c|c|c|c|c|c|c|c|c|c|c|c|c|c|c|c|c|c|}
\hline & \multirow[b]{2}{*}{ LRI $^{1}$} & \multirow[b]{2}{*}{$\mathbf{R I}^{2}$} & \multicolumn{4}{|c|}{$\mathbf{C}^{3}$} & \multicolumn{4}{|c|}{$\mathbf{R}$} & \multicolumn{4}{|c|}{$\mathbf{R Y}$} & \multirow[b]{2}{*}{ RMSE $^{4}$} & \multirow[b]{2}{*}{$P_{t}^{5}$} & \multirow[b]{2}{*}{$\boldsymbol{P}_{\mathrm{f}}$} \\
\hline & & & $60 \mathrm{~d}$ & & $83 \mathrm{~d}$ & & $60 \mathrm{~d}$ & & $83 \mathrm{~d}$ & & $60 \mathrm{~d}$ & & $83 \mathrm{~d}$ & & & & \\
\hline \multicolumn{18}{|l|}{ Microbial origin } \\
\hline Amino acid degradation & & & 155.29 & c & 202.53 & c & 135.14 & c & 210.42 & c & 457.22 & $\mathbf{a}$ & 355.54 & $\mathbf{b}$ & 51.70 & ns & $* * *$ \\
\hline Methanethiol & 473 & $\mathrm{a}$ & 28.43 & $\mathrm{c}$ & 60.35 & $\mathrm{a}$ & 30.51 & $\mathrm{c}$ & 64.79 & $\mathrm{a}$ & 43.02 & $\mathrm{~b}$ & 16.27 & $\mathrm{~d}$ & 5.59 & $* * *$ & $* * *$ \\
\hline 2-Methylpropanal & 593 & $\mathrm{a}$ & 7.36 & $\mathrm{~b}$ & 5.25 & $\mathrm{~b}$ & 5.76 & $\mathrm{~b}$ & 4.46 & $\mathrm{~b}$ & 26.54 & $\mathrm{a}$ & 5.78 & $\mathrm{~b}$ & 8.17 & $* *$ & $* *$ \\
\hline 3-Methylbutanal & 690 & $\mathrm{a}$ & 35.96 & $a b$ & 32.74 & $\mathrm{~b}$ & 32.29 & $\mathrm{~b}$ & 26.29 & $\mathrm{~b}$ & 55.26 & $\mathrm{a}$ & 24.05 & $\mathrm{~b}$ & 12.61 & $* *$ & ns \\
\hline 2-Methylbutanal (58) & 698 & $\mathrm{a}$ & 1.94 & $\mathrm{~b}$ & 1.73 & $\mathrm{~b}$ & 1.93 & $\mathrm{~b}$ & 1.61 & $\mathrm{~b}$ & 6.26 & $\mathrm{a}$ & 1.62 & $\mathrm{~b}$ & 1.72 & $* *$ & $* *$ \\
\hline Dimethyl disulfide & 773 & $\mathrm{a}$ & 18.21 & $\mathrm{a}$ & 5.73 & $\mathrm{~b}$ & 9.61 & $a b$ & 5.77 & $\mathrm{~b}$ & 4.66 & $\mathrm{~b}$ & 7.60 & $\mathrm{ab}$ & 6.14 & $*$ & ns \\
\hline Toluene (91) & 788 & $\mathrm{a}$ & 10.62 & $\mathrm{a}$ & 8.98 & $\mathrm{ab}$ & 8.14 & $a b$ & 7.04 & $\mathrm{~b}$ & 7.80 & $\mathrm{~b}$ & 6.79 & $\mathrm{~b}$ & 1.47 & $*$ & $* * *$ \\
\hline 3-Methyl-1-butanol (55) & 795 & $\mathrm{a}$ & 2.66 & $\mathrm{c}$ & 2.14 & $\mathrm{c}$ & 2.85 & $\mathrm{c}$ & 2.52 & $\mathrm{c}$ & 33.68 & $\mathrm{a}$ & 16.31 & $\mathrm{~b}$ & 1.78 & $* * *$ & $* * *$ \\
\hline 2-Methyl propanoic acid & 864 & $\mathrm{a}$ & 14.80 & $\mathrm{~b}$ & 32.68 & $\mathrm{~b}$ & 11.84 & $\mathrm{~b}$ & 48.07 & $\mathrm{~b}$ & 142.41 & $\mathrm{a}$ & 161.01 & $\mathrm{a}$ & 30.95 & $*$ & $* * *$ \\
\hline 3-Methyl butanoic acid (60) & 941 & $\mathrm{a}$ & ND & $\mathrm{b}$ & ND & $\mathrm{b}$ & ND & $\mathrm{b}$ & ND & $\mathrm{b}$ & 93.81 & $\mathrm{a}$ & 127.11 & $\mathrm{a}$ & 26.67 & ns & $* * *$ \\
\hline 2-Acetyl-1-pyrroline & 961 & $\mathrm{a}$ & $\operatorname{tr}^{6}$ & & $\operatorname{tr}$ & & $\operatorname{tr}$ & & $\operatorname{tr}$ & & $\operatorname{tr}$ & & $\operatorname{tr}$ & & & & \\
\hline 2,6-Dimethylpyrazine (108) & 944 & $\mathrm{a}$ & 2.88 & $\mathrm{~b}$ & 21.26 & $\mathrm{a}$ & 3.63 & $\mathrm{~b}$ & 18.85 & $\mathrm{a}$ & 0.54 & $\mathrm{~b}$ & 2.54 & $\mathrm{~b}$ & 4.11 & $* * *$ & $* * *$ \\
\hline 2-Methyl butanoic acid (74) & 946 & $\mathrm{a}$ & 6.54 & $\mathrm{~b}$ & 8.63 & $\mathrm{~b}$ & 8.59 & $\mathrm{~b}$ & 11.38 & $\mathrm{~b}$ & 29.36 & $\mathrm{a}$ & 29.98 & $\mathrm{a}$ & 5.46 & ns & $* * *$ \\
\hline 3-(Methylthio)propanal (104) & 968 & $\mathrm{a}$ & 1.45 & a & 1.06 & $a b$ & 0.79 & $\mathrm{bc}$ & 0.68 & bc & 0.33 & $\mathrm{c}$ & 0.49 & $\mathrm{c}$ & 0.27 & ns & $* * *$ \\
\hline Dimethyl trisulfide & 1003 & $\mathrm{a}$ & $\operatorname{tr}$ & & $\operatorname{tr}$ & & $\operatorname{tr}$ & & $\operatorname{tr}$ & & $\operatorname{tr}$ & & $\operatorname{tr}$ & & & & \\
\hline Benzeneacetaldehyde & 1110 & $\mathrm{a}$ & 10.93 & $\mathrm{a}$ & 9.99 & $\mathrm{ab}$ & 7.11 & $\mathrm{bc}$ & 7.89 & $a b c$ & 5.11 & $\mathrm{c}$ & 8.73 & $\mathrm{ab}$ & 1.84 & ns & $* * *$ \\
\hline Phenol & 1114 & $\mathrm{a}$ & 13.53 & $\mathrm{a}$ & 13.42 & $\mathrm{a}$ & 12.09 & $\mathrm{a}$ & 12.97 & $\mathrm{a}$ & 8.44 & $\mathrm{~b}$ & 10.91 & $\mathrm{ab}$ & 1.90 & ns & $* * *$ \\
\hline 2-Acetyl-2-thiazoline & 1175 & $\mathrm{a}$ & $\operatorname{tr}$ & & $\operatorname{tr}$ & & $\operatorname{tr}$ & & $\operatorname{tr}$ & & $\operatorname{tr}$ & & $\operatorname{tr}$ & & & & \\
\hline
\end{tabular}


Table 2. Continued.

\begin{tabular}{|c|c|c|c|c|c|c|c|c|c|c|c|c|c|c|c|c|c|}
\hline & \multirow[b]{2}{*}{ LRI $^{1}$} & \multirow[b]{2}{*}{$\mathbf{R I}^{2}$} & \multicolumn{4}{|c|}{$\mathbf{C}^{3}$} & \multicolumn{4}{|c|}{$\mathbf{R}$} & \multicolumn{4}{|c|}{ RY } & \multirow[b]{2}{*}{$\mathrm{RMSE}^{4}$} & \multirow[b]{2}{*}{$P_{t}^{5}$} & \multirow[b]{2}{*}{$\boldsymbol{P}_{\mathrm{f}}$} \\
\hline & & & $60 \mathrm{~d}$ & & $83 \mathrm{~d}$ & & $60 \mathrm{~d}$ & & $83 \mathrm{~d}$ & & $60 \mathrm{~d}$ & & $83 \mathrm{~d}$ & & & & \\
\hline Carbohydrate fermentation & & & 5631.62 & $\mathbf{b}$ & 7405.09 & $\mathbf{a}$ & 6280.08 & $\mathbf{a b}$ & 6877.28 & $\mathbf{a b}$ & 3473.90 & c & 3777.01 & c & 983.47 & $* *$ & $* * *$ \\
\hline Acetaldehyde & 467 & $\mathrm{a}$ & 25.14 & $\mathrm{~b}$ & 20.89 & $\mathrm{~b}$ & 18.13 & $\mathrm{~b}$ & 16.98 & $\mathrm{~b}$ & 73.09 & $\mathrm{a}$ & 28.18 & $\mathrm{~b}$ & 13.21 & $* * *$ & $* * *$ \\
\hline Ethyl alcohol & 507 & $\mathrm{a}$ & 887.72 & $\mathrm{~b}$ & 739.85 & $\mathrm{~b}$ & 670.60 & $\mathrm{~b}$ & 854.35 & $\mathrm{~b}$ & 2294.16 & a & 2192.11 & $\mathrm{a}$ & 151.08 & ns & $* * *$ \\
\hline Acetone & 529 & $\mathrm{a}$ & 69.34 & $\mathrm{a}$ & 47.78 & $\mathrm{ab}$ & 61.17 & $\mathrm{ab}$ & 42.92 & $\mathrm{~b}$ & 49.51 & $a b$ & 54.99 & $a b$ & 13.49 & $* *$ & ns \\
\hline 2,3-Butanedione & 627 & $\mathrm{a}$ & 129.85 & $\mathrm{a}$ & 129.93 & $\mathrm{a}$ & 160.14 & $\mathrm{a}$ & 119.13 & $\mathrm{a}$ & 19.36 & $\mathrm{~b}$ & 9.69 & $\mathrm{~b}$ & 27.37 & ns & $* * *$ \\
\hline 2-Butanone & 631 & $\mathrm{a}$ & 72.68 & $\mathrm{a}$ & 48.17 & $\mathrm{~b}$ & 79.28 & $\mathrm{a}$ & 43.59 & $\mathrm{~b}$ & 28.79 & $\mathrm{~b}$ & 26.80 & $\mathrm{~b}$ & 13.22 & $* * *$ & $* * *$ \\
\hline Acetic acid & 717 & $\mathrm{a}$ & 2805.24 & $\mathrm{~b}$ & 4435.95 & $\mathrm{a}$ & 3122.49 & $\mathrm{~b}$ & 3822.32 & $a b$ & 813.68 & $\mathrm{c}$ & 1343.73 & $\mathrm{c}$ & 651.28 & $* * *$ & $* * *$ \\
\hline 3-Hydroxy-2-butanone & 781 & $\mathrm{a}$ & 1586.48 & $\mathrm{a}$ & 1914.66 & $\mathrm{a}$ & 2109.15 & $\mathrm{a}$ & 1890.61 & $\mathrm{a}$ & 150.70 & $\mathrm{~b}$ & 46.42 & $\mathrm{~b}$ & 400.29 & ns & $* * *$ \\
\hline Butanoic acid & 891 & $\mathrm{a}$ & 55.16 & de & 67.85 & $\mathrm{bc}$ & 59.12 & $\mathrm{~cd}$ & 87.39 & $\mathrm{a}$ & 44.60 & $\mathrm{e}$ & 75.09 & $\mathrm{~b}$ & 6.81 & $* * *$ & $* * *$ \\
\hline Esterase activity & & & 404.73 & b & 364.80 & b & 415.44 & $\mathbf{b}$ & 423.13 & b & 1077.97 & $\mathbf{a}$ & 1134.48 & $\mathbf{a}$ & 112.28 & ns & $* * *$ \\
\hline Ethyl acetate & 635 & a & 229.96 & bc & 178.56 & $\mathrm{c}$ & 283.17 & $\mathrm{~b}$ & 199.47 & bc & 430.45 & a & 462.49 & a & 58.33 & ns & $* * *$ \\
\hline Ethyl 2-methylpropanoate (71) & 788 & $\mathrm{a}$ & ND & $\mathrm{b}$ & ND & $\mathrm{b}$ & ND & $\mathrm{b}$ & ND & $\mathrm{b}$ & 30.70 & $\mathrm{a}$ & 31.56 & $\mathrm{a}$ & 4.13 & ns & $* * *$ \\
\hline Ethyl butanoate & 831 & $\mathrm{a}$ & 29.65 & $\mathrm{~b}$ & 29.57 & $\mathrm{~b}$ & 27.13 & $\mathrm{~b}$ & 32.82 & $\mathrm{~b}$ & 69.91 & $\mathrm{a}$ & 77.54 & $\mathrm{a}$ & 7.21 & ns & $* * *$ \\
\hline Ethyl 2-hydroxypropanoate & 867 & $\mathrm{a}$ & 40.96 & $\mathrm{bc}$ & 46.99 & $\mathrm{c}$ & 26.86 & $\mathrm{~d}$ & 51.52 & $\mathrm{c}$ & 81.60 & $\mathrm{~b}$ & 143.68 & $\mathrm{a}$ & 10.39 & $* * *$ & $* * *$ \\
\hline Ethyl 2-methylbutanoate (57) & 878 & $\mathrm{a}$ & 2.41 & $\mathrm{~b}$ & 3.26 & $\mathrm{~b}$ & 2.60 & $\mathrm{~b}$ & 5.20 & $\mathrm{~b}$ & 31.95 & $\mathrm{a}$ & 32.78 & $\mathrm{a}$ & 5.84 & ns & $* * *$ \\
\hline Ethyl 3-methylbutanoate & 882 & $\mathrm{a}$ & 33.96 & $\mathrm{~b}$ & 55.91 & $\mathrm{~b}$ & 32.89 & $\mathrm{~b}$ & 94.84 & $\mathrm{~b}$ & 387.96 & a & 386.12 & $\mathrm{a}$ & 84.17 & ns & $* * *$ \\
\hline 3-Methylbutyl acetate (70) & 907 & $\mathrm{a}$ & ND & $\mathrm{b}$ & ND & $\mathrm{b}$ & ND & $\mathrm{b}$ & ND & $\mathrm{b}$ & 1.00 & $\mathrm{a}$ & 1.03 & $\mathrm{a}$ & 0.10 & ns & $* * *$ \\
\hline Ethyl pentanoate & 928 & $\mathrm{a}$ & 21.53 & $\mathrm{a}$ & 12.56 & $\mathrm{bc}$ & 13.65 & $\mathrm{~b}$ & 10.93 & bcd & 8.44 & $\mathrm{~cd}$ & 6.76 & $d$ & 2.82 & $* * *$ & $* * *$ \\
\hline Ethyl hexanoate & 1030 & $\mathrm{a}$ & 45.43 & $\mathrm{a}$ & 37.45 & $a b$ & 28.48 & $\mathrm{~b}$ & 27.88 & $\mathrm{~b}$ & 32.58 & $\mathrm{~b}$ & 36.30 & $a b$ & 6.71 & ns & $* * *$ \\
\hline Ethyl octanoate (88) & 1230 & $\mathrm{a}$ & 0.83 & $\mathrm{c}$ & 0.50 & $\mathrm{c}$ & 0.66 & $\mathrm{c}$ & 0.46 & $\mathrm{c}$ & 3.37 & $\mathrm{~b}$ & 4.10 & $\mathrm{a}$ & 0.28 & ns & $* * *$ \\
\hline
\end{tabular}


Table 2. Continued.

\begin{tabular}{|c|c|c|c|c|c|c|c|c|c|c|c|c|c|c|c|c|c|}
\hline \multirow[b]{3}{*}{$\beta$-Oxidation } & \multirow[b]{2}{*}{$\mathbf{L R I}^{1}$} & \multirow[b]{2}{*}{$\mathbf{R I}^{2}$} & \multicolumn{4}{|c|}{$\mathbf{C}^{3}$} & \multicolumn{4}{|c|}{$\mathbf{R}$} & \multicolumn{4}{|c|}{ RY } & \multirow[b]{2}{*}{$\mathbf{R M S E}^{4}$} & \multirow[b]{2}{*}{$P_{t}^{5}$} & \multirow[b]{2}{*}{$\boldsymbol{P}_{\mathrm{f}}$} \\
\hline & & & \multicolumn{2}{|l|}{$60 \mathrm{~d}$} & \multicolumn{2}{|l|}{$83 \mathrm{~d}$} & \multirow[t]{2}{*}{$60 \mathrm{~d}$} & \multicolumn{3}{|c|}{$83 \mathrm{~d}$} & \multicolumn{2}{|l|}{$60 \mathrm{~d}$} & \multicolumn{2}{|l|}{$83 \mathrm{~d}$} & & & \\
\hline & & & & & & & & & & & & & & & & & \\
\hline 1-Octen-3-ol & 1032 & $\mathrm{a}$ & 30.49 & $\mathrm{a}$ & 29.09 & $\mathrm{a}$ & 25.23 & $\mathrm{a}$ & 24.13 & $\mathrm{a}$ & 10.04 & $\mathrm{~b}$ & 12.43 & $\mathrm{~b}$ & 5.00 & ns & $* * *$ \\
\hline \multicolumn{18}{|l|}{ Chemical origin } \\
\hline Lipid oxidation & & & 3441.38 & $\mathbf{a}$ & 2502.57 & $\mathbf{b}$ & 1768.25 & bc & 1192.48 & cd & 539.38 & d & 603.53 & d & 499.39 & $* *$ & $* * *$ \\
\hline Pentane & 500 & $\mathrm{a}$ & 31.95 & & 17.41 & & 28.03 & & 28.22 & & 32.05 & & 35.80 & & 11.09 & ns & $\mathrm{ns}$ \\
\hline Hexane & 600 & $\mathrm{a}$ & 8.28 & & 5.01 & & 8.38 & & 7.40 & & 9.35 & & 7.43 & & 2.62 & $*$ & ns \\
\hline 1-Propanol & 312 & $\mathrm{a}$ & 2.02 & $\mathrm{c}$ & 1.99 & $\mathrm{c}$ & 1.95 & $\mathrm{c}$ & 1.74 & $\mathrm{c}$ & 21.69 & $\mathrm{a}$ & 13.16 & $\mathrm{~b}$ & 1.66 & $* * *$ & $* * *$ \\
\hline Butanal & 622 & $\mathrm{a}$ & 3.68 & $\mathrm{a}$ & 2.41 & $\mathrm{~b}$ & 1.91 & $\mathrm{bc}$ & 1.51 & bcd & 0.77 & $\mathrm{~d}$ & 1.22 & $\mathrm{~cd}$ & 0.62 & ns & $* * *$ \\
\hline Heptane & 700 & $\mathrm{a}$ & 9.49 & & 6.94 & & 9.76 & & 8.63 & & 6.92 & & 7.06 & & 3.20 & ns & $\mathrm{ns}$ \\
\hline Pentanal & 739 & $\mathrm{a}$ & 136.60 & $\mathrm{a}$ & 88.75 & $\mathrm{~b}$ & 69.27 & $\mathrm{bc}$ & 49.72 & bcd & 19.23 & $\mathrm{~d}$ & 31.16 & $\mathrm{~cd}$ & 24.34 & $*$ & $* * *$ \\
\hline Octane & 800 & a & 135.27 & $\mathrm{a}$ & 128.26 & $\mathrm{ab}$ & 120.72 & $a b c$ & 102.54 & $\mathrm{abc}$ & 74.20 & $\mathrm{c}$ & 82.53 & $\mathrm{bc}$ & 29.58 & ns & $* * *$ \\
\hline Propanoic acid & 811 & $\mathrm{a}$ & 14.80 & $\mathrm{bc}$ & 21.55 & $\mathrm{a}$ & 12.39 & $\mathrm{c}$ & 18.80 & $a b$ & 12.72 & $\mathrm{c}$ & 15.09 & $\mathrm{bc}$ & 2.77 & $* * *$ & $* *$ \\
\hline 1-Pentanol (70) & 827 & $\mathrm{a}$ & 4.45 & $\mathrm{a}$ & 2.57 & $\mathrm{~b}$ & 3.83 & $\mathrm{a}$ & 2.02 & $\mathrm{bc}$ & 1.82 & $\mathrm{bc}$ & 1.15 & $\mathrm{c}$ & 0.65 & $* * *$ & $* * *$ \\
\hline Hexanal & 842 & $\mathrm{a}$ & 2774.14 & $\mathrm{a}$ & 1969.97 & $\mathrm{~b}$ & 1223.18 & $\mathrm{c}$ & 768.59 & $\mathrm{~cd}$ & 201.53 & $\mathrm{~d}$ & 270.65 & $d$ & 410.63 & $* *$ & $* * *$ \\
\hline 1-Hexanol & 924 & a & 81.87 & $\mathrm{a}$ & 39.79 & $\mathrm{ab}$ & 80.92 & $\mathrm{ab}$ & 37.78 & $\mathrm{bc}$ & 30.23 & $\mathrm{c}$ & 11.20 & $\mathrm{c}$ & 24.98 & $* * *$ & $* * *$ \\
\hline Heptanal (70) & 941 & a & 17.36 & $\mathrm{a}$ & 17.18 & $a b$ & 13.11 & $a b$ & 8.69 & $\mathrm{~b}$ & ND & $\mathrm{b}$ & ND & $\mathrm{b}$ & 5.62 & ns & $* *$ \\
\hline Pentanoic acid (60) & 980 & $\mathrm{a}$ & 4.00 & $a b$ & 4.96 & $\mathrm{a}$ & 3.29 & $\mathrm{~b}$ & 4.23 & $a b$ & 1.08 & $\mathrm{c}$ & 1.30 & $\mathrm{c}$ & 0.73 & $* *$ & $* * *$ \\
\hline 2-Pentylfuran & 1010 & $\mathrm{a}$ & 25.20 & $\mathrm{a}$ & 25.22 & $\mathrm{a}$ & 15.42 & $\mathrm{~b}$ & 17.78 & $\mathrm{~b}$ & 3.79 & $\mathrm{c}$ & 4.28 & $\mathrm{c}$ & 3.51 & ns & $* * *$ \\
\hline (Z)- 2-Heptenal & 1012 & a & 10.45 & $\mathrm{a}$ & 8.20 & $a b$ & 5.90 & $\mathrm{bc}$ & 5.65 & $\mathrm{c}$ & 1.89 & & ND & & 1.47 & $*$ & $* * *$ \\
\hline Octanal (56) & 1049 & $\mathrm{a}$ & 7.14 & $\mathrm{a}$ & 6.27 & $\mathrm{a}$ & 5.71 & $\mathrm{ab}$ & 3.71 & $\mathrm{bc}$ & 3.02 & $\mathrm{c}$ & 3.12 & $\mathrm{c}$ & 1.33 & $*$ & $* * *$ \\
\hline Hexanoic acid (60) & 1077 & $\mathrm{a}$ & 22.26 & $\mathrm{a}$ & 25.95 & $\mathrm{a}$ & 19.09 & $\mathrm{a}$ & 22.42 & $\mathrm{a}$ & 5.27 & $\mathrm{~b}$ & 6.88 & $\mathrm{~b}$ & 3.98 & 0.038 & $* * *$ \\
\hline (E)-2-Octenal & 1117 & $\mathrm{a}$ & 13.03 & $\mathrm{a}$ & 11.01 & $\mathrm{ab}$ & 7.45 & $\mathrm{bc}$ & 7.76 & $\mathrm{bc}$ & 1.37 & & 5.17 & $\mathrm{~cd}$ & 2.25 & ns & $* * *$ \\
\hline Nonanal & 1151 & $\mathrm{a}$ & 122.97 & & 95.75 & & 122.58 & & 75.31 & & 97.86 & & 87.20 & & 30.58 & $* *$ & $\mathrm{~ns}$ \\
\hline
\end{tabular}


Table 2. Continued.

\begin{tabular}{|c|c|c|c|c|c|c|c|c|c|c|c|c|c|c|}
\hline & \multirow[b]{2}{*}{ LRI $^{1}$} & \multirow[b]{2}{*}{$\mathbf{R I}^{2}$} & \multicolumn{4}{|c|}{$\mathbf{C}^{3}$} & \multicolumn{2}{|c|}{$\mathbf{R}$} & \multicolumn{3}{|c|}{ RY } & \multirow[b]{2}{*}{ RMSE $^{4}$} & \multirow[b]{2}{*}{$P_{t}^{5}$} & \multirow[b]{2}{*}{$\boldsymbol{P}_{\mathrm{f}}$} \\
\hline & & & $60 \mathrm{~d}$ & & $83 \mathrm{~d}$ & & $60 \mathrm{~d}$ & $83 \mathrm{~d}$ & $60 \mathrm{~d}$ & & $83 \mathrm{~d}$ & & & \\
\hline (E)-2-Nonenal & 1224 & $\mathrm{a}$ & 3.69 & $\mathrm{a}$ & 3.59 & $\mathrm{a}$ & $2.36 \mathrm{~b}$ & $2.15 \mathrm{~b}$ & 1.11 & $\mathrm{c}$ & $1.92 \mathrm{bc}$ & 0.49 & ns & $* * *$ \\
\hline Octanoic acid & 1267 & $\mathrm{a}$ & 11.02 & $\mathrm{~d}$ & 18.10 & $\mathrm{a}$ & $11.42 \mathrm{~cd}$ & $16.30 \mathrm{ab}$ & 12.29 & bcd & $16.16 \mathrm{abc}$ & 2.76 & $* * *$ & ns \\
\hline Nonanoic acid (60) & 1359 & $\mathrm{a}$ & 1.70 & & 1.70 & & 1.56 & 1.53 & 1.51 & & 1.03 & 0.96 & ns & $\mathrm{ns}$ \\
\hline \multicolumn{15}{|l|}{ Thiamine degradation } \\
\hline 2-Methyl-3-furanthiol & 896 & $\mathrm{a}$ & $\operatorname{tr}$ & & $\operatorname{tr}$ & & $\operatorname{tr}$ & $\operatorname{tr}$ & $\operatorname{tr}$ & & $\operatorname{tr}$ & & & \\
\hline Methyl-2-methyl-3-furyldisulfide & 1221 & $\mathrm{a}$ & $\operatorname{tr}$ & & $\operatorname{tr}$ & & $\operatorname{tr}$ & $\operatorname{tr}$ & $\operatorname{tr}$ & & $\operatorname{tr}$ & & & \\
\hline Spices & & & 3105.15 & & 3210.12 & & 3087.19 & 2804.85 & 3299.25 & & 2941.91 & 376.00 & ns & ns \\
\hline O-Xylene (91) & 917 & $\mathrm{a}$ & 0.84 & & 0.72 & & 0.70 & 0.67 & 0.69 & & 0.59 & 0.14 & ns & ns \\
\hline Styrene (104) & 919 & $\mathrm{a}$ & 0.57 & $\mathrm{c}$ & 0.78 & $\mathrm{c}$ & $0.51 \mathrm{c}$ & $0.64 \mathrm{c}$ & 3.17 & $\mathrm{a}$ & $2.53 \mathrm{~b}$ & 0.19 & ns & $* * *$ \\
\hline Unidentified terpene & 934 & $\mathrm{c}$ & 165.77 & & 141.31 & & 168.37 & 138.55 & 160.73 & & 141.74 & 20.13 & $* * *$ & ns \\
\hline$\beta$-Phellandrene & 988 & $\mathrm{~b}$ & 460.31 & & 419.40 & & 486.90 & 407.33 & 490.25 & & 440.91 & 66.65 & $*$ & $\mathrm{~ns}$ \\
\hline$\beta$-Myrcene (93) & 1003 & $\mathrm{a}$ & 29.80 & & 33.77 & & 29.68 & 28.42 & 33.01 & & 29.16 & 4.26 & ns & ns \\
\hline Unidentified terpene & 1019 & $\mathrm{c}$ & 113.30 & & 121.94 & & 120.30 & 105.53 & 126.23 & & 110.98 & 19.27 & ns & $\mathrm{ns}$ \\
\hline 3-Carene & 1022 & $\mathrm{a}$ & 571.43 & & 630.26 & & 555.16 & 515.62 & 638.08 & & 540.43 & 100.34 & ns & ns \\
\hline Unidentified terpene & 1034 & $\mathrm{c}$ & 51.50 & $\mathrm{ab}$ & 48.03 & $\mathrm{~b}$ & $55.76 \mathrm{ab}$ & $48.49 \mathrm{~b}$ & 61.38 & $\mathrm{a}$ & $55.69 \mathrm{ab}$ & 6.19 & $*$ & $* *$ \\
\hline D-limonene & 1046 & $\mathrm{a}$ & 1081.58 & & 1170.91 & & 1063.91 & 1004.14 & 1133.01 & & 1022.63 & 129.31 & ns & ns \\
\hline Unidentified terpene & 1075 & $\mathrm{c}$ & 68.52 & & 68.29 & & 68.15 & 61.41 & 83.39 & & 66.98 & 17.45 & ns & ns \\
\hline Unidentified terpene (93) & 1099 & $\mathrm{c}$ & 0.99 & & 0.98 & & 0.80 & 0.81 & 0.90 & & 0.78 & 0.14 & ns & ns \\
\hline Terpinolene (93) & 1101 & $\mathrm{a}$ & 3.70 & & 3.86 & & 3.75 & 3.31 & 4.08 & & 3.48 & 0.50 & ns & ns \\
\hline Unidentified terpene & 1121 & $\mathrm{c}$ & 15.05 & $\mathrm{ab}$ & 19.32 & $\mathrm{a}$ & $14.83 \mathrm{ab}$ & $17.49 \mathrm{ab}$ & 14.48 & $\mathrm{~b}$ & $19.14 \mathrm{a}$ & 2.57 & $* * *$ & ns \\
\hline Unidentified terpene & 1366 & $\mathrm{c}$ & 26.89 & $\mathrm{a}$ & 26.61 & $\mathrm{a}$ & $22.58 \mathrm{ab}$ & $20.12 b$ & 23.74 & $a b$ & $21.74 \mathrm{ab}$ & 3.62 & ns & $* *$ \\
\hline Unidentified terpene & 1377 & $\mathrm{c}$ & 8.24 & & 9.49 & & 6.77 & 7.60 & 7.66 & & 8.29 & 2.50 & ns & ns \\
\hline Unidentified terpene & 1407 & $\mathrm{c}$ & 71.50 & $a b$ & 77.51 & $\mathrm{a}$ & $64.64 \mathrm{ab}$ & $59.95 \mathrm{~b}$ & 66.96 & $a b$ & $64.48 \mathrm{ab}$ & 9.45 & ns & $* *$ \\
\hline Caryophyllene & 1470 & $\mathrm{a}$ & 435.18 & & 436.96 & & 424.39 & 384.77 & 451.48 & & 412.34 & 49.79 & ns & ns \\
\hline
\end{tabular}


Table 2. Continued.

\begin{tabular}{|c|c|c|c|c|c|c|c|c|c|c|c|c|c|c|}
\hline & \multirow[b]{2}{*}{ LRI $^{1}$} & \multirow[b]{2}{*}{$\mathbf{R I}^{2}$} & \multicolumn{3}{|c|}{$\mathbf{C}^{3}$} & \multicolumn{2}{|c|}{$\mathbf{R}$} & \multicolumn{4}{|c|}{$\mathbf{R Y}$} & \multirow[b]{2}{*}{$\mathrm{RMSE}^{4}$} & \multirow[b]{2}{*}{$P_{t}^{5}$} & \multirow[b]{2}{*}{$\boldsymbol{P}_{\mathrm{f}}$} \\
\hline & & & $60 \mathrm{~d}$ & & $83 \mathrm{~d}$ & $60 \mathrm{~d}$ & $83 \mathrm{~d}$ & $60 \mathrm{~d}$ & & $83 \mathrm{~d}$ & & & & \\
\hline Unknown origin & & & 27.74 & $\mathbf{b}$ & 29.19 c & 34.36 ab & $39.25 \mathrm{a}$ & 63.34 & $\mathbf{a b}$ & 64.12 & $\mathbf{a b}$ & 13.80 & ns & $* * *$ \\
\hline 2-Methyl-1-propene (41) & 459 & $\mathrm{~b}$ & 4.87 & $\mathrm{a}$ & $4.08 \mathrm{ab}$ & $2.80 \mathrm{bc}$ & $2.13 \mathrm{c}$ & 1.42 & $\mathrm{c}$ & 1.61 & $\mathrm{c}$ & 0.88 & ns & $* * *$ \\
\hline Carbon disulfide & 537 & $\mathrm{a}$ & 21.97 & $\mathrm{~b}$ & $23.95 \mathrm{~b}$ & $30.58 \mathrm{~b}$ & $36.01 \mathrm{~b}$ & 66.95 & $\mathrm{a}$ & 61.37 & $\mathrm{a}$ & 13.87 & ns & $* * *$ \\
\hline 4-Methylphenol (107) & 1197 & $\mathrm{a}$ & 0.90 & $\mathrm{~b}$ & $1.16 \mathrm{a}$ & $0.98 \mathrm{~b}$ & $1.11 \mathrm{a}$ & 0.97 & $\mathrm{~b}$ & 1.14 & $\mathrm{a}$ & 0.07 & $* * *$ & ns \\
\hline
\end{tabular}

${ }^{1} \mathrm{LRI}$ : Linear retention index of the compounds eluted from the GC-MS using a DB-624 capillary column. ${ }^{2}$ RI: Reliability of identification: a, identification by mass spectrum and by coincidence with LRI of authentic standard; $b$, tentative identification by mass spectrum; c: unknown. ${ }^{3} \mathrm{C}$ : control batch; R: $47 \%$ reduction in nitrite and nitrate; RY: $47 \%$ reduction in nitrite and nitrate and $D$. hansenii inoculated. ${ }^{4} \mathrm{RMSE}$ : Roost mean square error. ${ }^{5} P_{\mathrm{t}}: P$ value of ripening time effect, $P_{\mathrm{f}}: P$ value of formulation effect. Different letters in the same row indicate significant differences at $* * * \mathrm{P}<0.001,{ }^{* *} \mathrm{P}<0.01, * \mathrm{P}<0.05$. ns: $\mathrm{P}>$ 0.05. ${ }^{6}$ tr: traces, not quantified. 


\subsection{Aroma characteristics of the sausages}

Gas chromatography-olfactometry (Table 3) revealed the presence of 29 aroma active zones which were identified except for 1 odour zone attributed to an identified terpene. The most important aroma compounds with the highest DF (detection frequency) values (DF $>10$ ) were 2,3-butanedione, acetic acid, hexanal, ethyl 2-methylbutanoate, 1-hexanol, 2-acetyl-1-pyrroline, methional, dimethyl trisulfide and 3-carene. The assessors were able to detect five odour zones that corresponded to compounds 2-methyl-3-furanthiol, 2-acetyl-1-pyrroline, dimethyl trisulfide, 2-acetyl-2-thiazoline and methyl-2-methyl-3-furyldisulfide which were not analysed by mass spectra due to their presence as trace levels.

The relationship between the instrumental variables and the ingoing amounts of nitrate and nitrite and $D$. hansenii inoculation at each ripening time, were examined by a principal component analysis (Figure 3). Comparisons were done using the parameters (physicochemical, microbiological parameters and aroma compounds) measurements at 60 (Fig. $3 \mathrm{~A}$ and B) and 83 d (Fig. $3 \mathrm{C}$ and $\mathrm{D}$ ) of ripening. Regarding results at $60 \mathrm{~d}$ (Fig. 3A), the first two principal components were able to explain the $72.09 \%$ of the total variance. PC1 accounted for $56.34 \%$ of the variance and distinguished sausages by $D$. hansenii inoculation, placing $\mathrm{C}$ and $\mathrm{R}$ sausages on the left quadrant and RY sausages on the right quadrant. Sausages inoculated with $D$. hansenii RY, were associated with higher water activity, $\mathrm{pH}$, yeast and moulds and colour parameters. Regarding aroma compounds, RY sausages were related to those derived from esterase activity, as well as 3-methylbutanal and methanethiol resulting from amino acid degradation. On the other hand, $\mathrm{C}$ and $\mathrm{R}$ sausages were related to an increase in TBARS and aroma compounds derived from lipid oxidation, $\beta$-oxidation and carbohydrate fermentation, as well as methional resulting from amino acid degradation. PC2 represented $15.76 \%$ of the variance corresponding to the variability among replicates.

Regarding results at $83 \mathrm{~d}$ of drying (Fig. $3 \mathrm{C}$ and D), two principal components were able to explain the $70.40 \%$ of the total variance. PC1 accounted for $56.21 \%$ of the variance and distinguished sausages by $D$. hansenii inoculation and nitrate and nitrite reduction, placing $C$ sausages on the left quadrant, R sausages on the centre and RY sausages on the right quadrant. Sausages inoculated with $D$. hansenii RY, were again related to higher water activity, yeast and moulds, colour parameters and aroma compounds derived from esterase activity. Moreover, $\mathrm{C}$ sausages were again related to TBARS and aroma compounds derived from lipid oxidation, while the R sausages were related to GCN counts and aroma compounds derived from amino acid degradation and carbohydrate fermentation. PC2 represented $14.19 \%$ of the variance corresponding to the variability among replicates. 
Table 3. Odour active compounds identified in dry fermented sausages.

\begin{tabular}{|c|c|c|c|c|c|}
\hline Compounds & $\begin{array}{l}\text { IRL } \\
\text { GC-O }\end{array}$ & $\begin{array}{l}\text { IRL std } \\
\text { GC-O }\end{array}$ & $\begin{array}{l}\mathbf{R I} \\
\end{array}$ & GC-O description & $\begin{array}{l}\text { DF } \\
\end{array}$ \\
\hline Methanethiol & 474 & 471 & $\mathrm{a}$ & Rotten, unpleasant & 9 \\
\hline 1-Propanol & 611 & 614 & $\mathrm{a}$ & Acid, fermented & 4 \\
\hline 2-Butanone & 628 & 632 & $\mathrm{a}$ & Wet, fresh, sweet & 3 \\
\hline 2,3-Butanedione & 633 & 638 & $\mathrm{a}$ & Cheese, butter, dairy, fruit & 11 \\
\hline 3-Methylbutanal & 690 & 691 & $\mathrm{a}$ & Sweet, green, spicy & 5 \\
\hline Acetic acid & 701 & 700 & $\mathrm{a}$ & Acid, fermented, vinegar & 11 \\
\hline 3-Hydroxy-2-butanone & 779 & 777 & $\mathrm{a}$ & Cardboard, green & 3 \\
\hline Ethyl 2-methylpropanoate & 783 & 789 & $\mathrm{a}$ & Sweet, fruity & 4 \\
\hline Ethyl butanoate & 824 & 825 & $\mathrm{a}$ & Sweet, fruity, fresh & 5 \\
\hline Hexanal & 835 & 836 & $\mathrm{a}$ & Green, fresh cut grass, fatty & 12 \\
\hline Ethyl 2-hydroxypropanoate & 861 & 859 & $\mathrm{a}$ & Fresh, floral, acid & 3 \\
\hline Ethyl 2-methylbutanoate & 872 & 872 & $\mathrm{a}$ & Pineapple, sweet, acid, unpleasant, & 10 \\
\hline Ethyl 3-methylbutanoate & 875 & 876 & $\mathrm{a}$ & Sweet, fruity, unpleasant, acid, & 7 \\
\hline 2-Methyl-3-furanthiol & 899 & 897 & $\mathrm{a}$ & Fatty, medicinal, unpleasant, sulfur & 3 \\
\hline 1-Hexanol & 925 & 919 & $\mathrm{a}$ & Fatty, rancid, rotten fruit, unpleasant & 12 \\
\hline Heptanal & 942 & 939 & $\mathrm{a}$ & Cured, rancid, unpleasant & 6 \\
\hline 2-Acetyl-1-pyrroline & 962 & 963 & $\mathrm{a}$ & $\begin{array}{l}\text { Roasted, fried corn, roasted nuts, } \\
\text { Cooked potatoes, vegetable, meaty, }\end{array}$ & 11 \\
\hline Methional & 969 & 969 & $\mathrm{a}$ & sulfur & 11 \\
\hline$\beta$-Myrcene & 1002 & 1003 & $\mathrm{a}$ & Green & 3 \\
\hline Dimethyl trisulfide & 1011 & 1009 & $\mathrm{a}$ & Spicy, pungent, sulfur, rotten, vegetable & 11 \\
\hline 3-Carene & 1021 & 1026 & $\mathrm{a}$ & Mushrooms, earthy, green, fresh & 12 \\
\hline 1-Octen-3-ol & 1032 & 1028 & $\mathrm{a}$ & Green, fresh, mushrooms & 5 \\
\hline Octanal & 1046 & 1047 & $\mathrm{a}$ & Sweet, citrus, floral & 7 \\
\hline Hexanoic acid & 1068 & 1064 & $\mathrm{a}$ & Cheese, unpleasant, floral fresh & 3 \\
\hline Unidentified terpene & 1092 & & $\mathrm{~b}$ & Spicy, fresh, floral & 3 \\
\hline Nonanal & 1150 & 1151 & $\mathrm{a}$ & $\begin{array}{l}\text { Fresh, herbaceous, green, unpleasant } \\
\text { Toasted, fried corn, caramel, toasted }\end{array}$ & 7 \\
\hline 2-Acetyl-2-thiazoline & 1180 & 1178 & $\mathrm{a}$ & corn, bread & 8 \\
\hline $\begin{array}{l}\text { 4-Methylphenol } \\
\text { Methyl-2-methyl-3-furyl } \\
\text { disulfide }\end{array}$ & 1198 & 1190 & $\mathrm{a}$ & $\begin{array}{l}\text { Unpleasant, rotten, sulphur } \\
\text { Meaty, unpleasant, wet wood, } \\
\text { fermented, rotten }\end{array}$ & 6 \\
\hline \multicolumn{6}{|c|}{$\begin{array}{l}{ }^{1} \text { Linear retention index of the compounds eluted from the GC-FID-O using a DB-624 capillary } \\
\text { column. } \\
{ }^{2} \text { Linear retention index of standard compounds in the GC-FID-O. } \\
{ }^{3} \text { Reliability of identification: a, identification by mass spectrum, coincidence with LRI of an } \\
\text { authentic standard and by coincidence of the assesors's descriptors with those described in the } \\
\text { Fenaroli's handbook of flavour ingredients (Burdok, 2002); b, tentative identification by mass } \\
\text { spectrum. } \\
\text { }{ }^{4} \text { Detection frequency value. }\end{array}$} \\
\hline
\end{tabular}


F1 and F2: $72.09 \%$

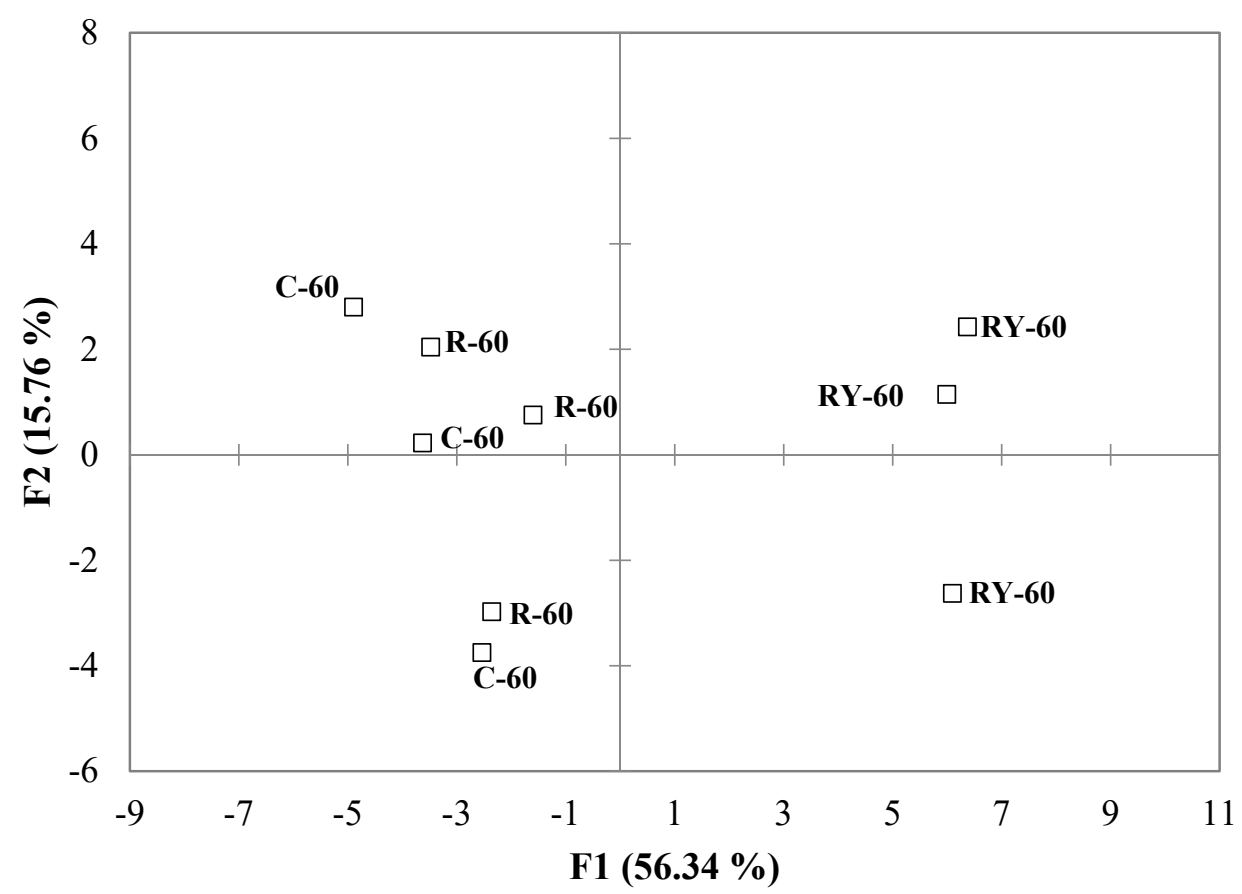

F1 and F2: $72.09 \%$

B

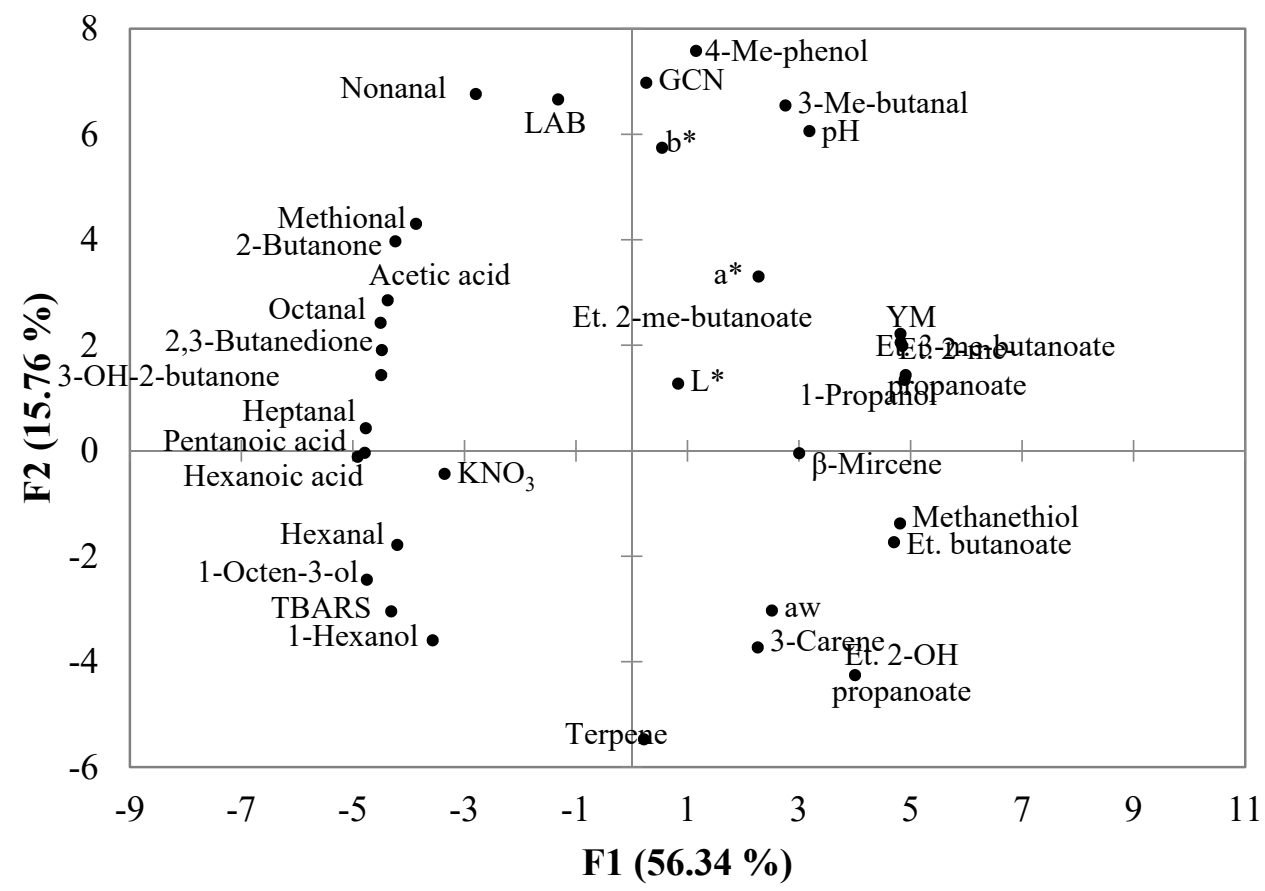

Figure 3. Loadings of the first two principal components (PC1-PC2) representing the variability (physicochemical, microbiological parameters and aroma compounds) of the three batches of dry fermented sausages at $60 \mathrm{~d}(\mathrm{~A}$ and $\mathrm{B})$ and $83 \mathrm{~d}(\mathrm{C}$ and $\mathrm{D})$ of ripening. $\mathrm{C}$ (control), $\mathrm{R}(47 \%$ reduction of nitrate and nitrite), RY (47\% reduction of nitrate and nitrite and inoculated with $D$. hansenii). YM: yeasts and moulds; LAB: lactic acid bacteria; GCN: Gram positive coagulase negative cocci. 
F1 and F2: $70.40 \%$

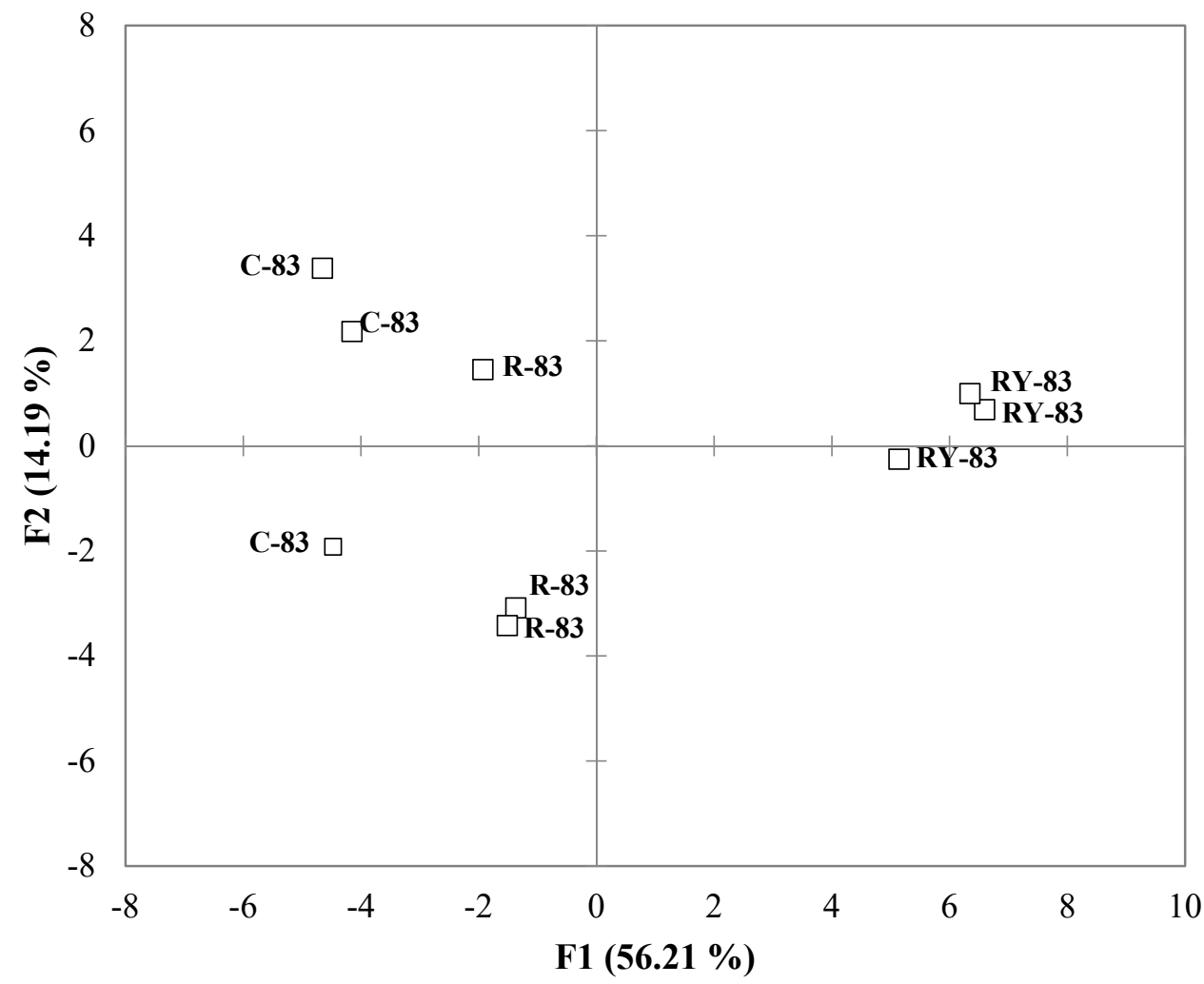

F1 and F2: $70.40 \%$

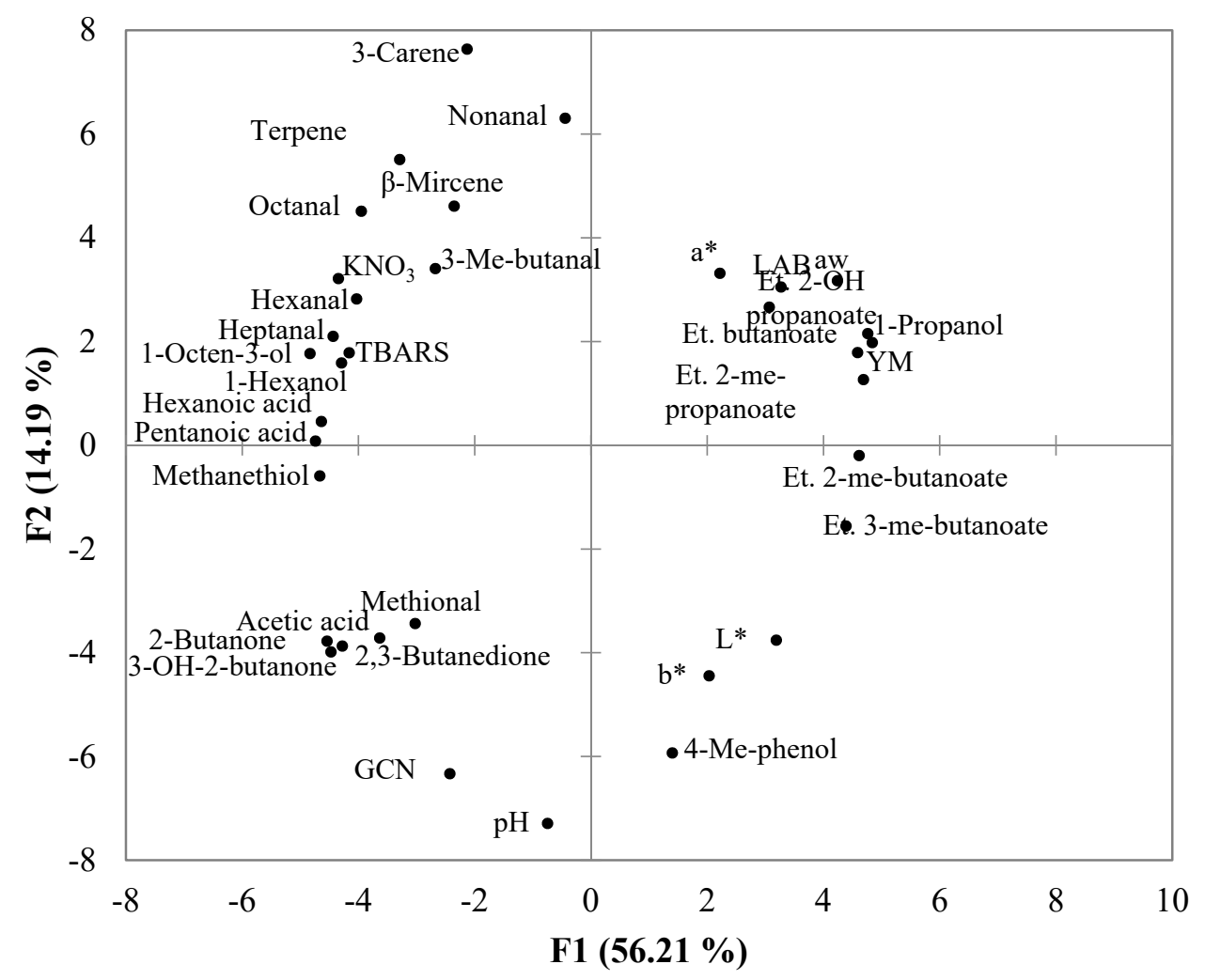

Figure 3. Continued. 


\section{Discussion}

Physicochemical changes observed were similar to the ones reported for this type of product (Olivares et al., 2010; Perea-Sanz et al., 2018) (Table 1 and Fig. 1). The reduction in the ingoing amounts of nitrate and nitrite was confirmed. The lowest nitrate reduction observed during drying in $\mathrm{C}$ sausages respect to $\mathrm{R}$ and $\mathrm{RY}$ sausages may be due to the generation of nitrate from nitrite oxidation (Honikel, 2008). Moreover, other authors have shown that the use of nitrate and nitrite mixtures increases the amount of residual nitrate (Gratacós-Cubarsí et al., 2013). The lowest residual nitrate detected in inoculated sausages (RY) could be explained by the inhibition of nitrite oxidation by $D$. hansenii (Cano-Garcia et al., 2014).

Nitrate and nitrite reduction did not affect $\mathrm{pH}$ value however, Aquilani et al. (2018) and Gonzales-Barron et al. (2015) observed a small increase in $\mathrm{pH}$ values due to the absence of nitrate and/or nitrite in dry fermented sausages. These differences among studies can be due to differences in formulations as well as differences in process manufacture like long drying times that may cause a $\mathrm{pH}$ increase. In RY sausages, $D$. hansenii consumption of organic acids and ammonium production (Flores, Corral, Cano-García, Salvador \& Belloch, 2015) increased pH at the end of the ripening.

Regarding lipid oxidation, a decrease in TBARS in R sausages was observed in previous studies (Perea-Sanz et al., 2018). However, this is not in agreement with the reported antioxidant ability of nitrite (Zanardi, Ghidini, Battaglia \& Chizzolini, 2004). Similar reductions in TBARS were observed in nitrite reduced Pastrima although they were related to the stage of processing (Aksu, Erdemir \& Çakici, 2016). The chemistry of nitrate and nitrite in cured meats is a complex subject (Majou \& Christieans, 2018). The nitric oxide generated from nitrite can react with neutrophil-derived superoxide present in muscle tissues and generate peroxynitrite $\left(\mathrm{ONOO}^{-}\right)$, which has prooxidant activity and antimicrobial effects (Brannan, Connolly \& Decker, 2001). Thus, in fermented sausages with high ingoing amounts of nitrate and nitrite, peroxynitrite formation might increase oxidation events including lipid oxidation. The nitrite prooxidant effect of peroxinitrite is directly related with its antimicrobial effect which is more harmful against Gram positive anaerobic bacteria, like C. botulinum (Majou \& Christieans, 2018). On the contrary, the antioxidant capacity of $D$. hansenii limits lipid oxidation processes as shown by its great antioxidant capacity (Corral et al., 2015) during the entire drying time and, moreover, prevents nitrite oxidation as observed by the lowest residual nitrate content in RY.

On the other hand, the reduction of the ingoing amounts of nitrate and nitrite did not have effect on the microbial counts. LAB counts at both drying times were stable as reported by Christieans et al. (2018), and GCN and yeasts and moulds counts (Fig. 1A, 1C) were comparable to those found by Cano-García et al. (2014). On the contrary, Hospital et al. (2015) reported an increase 
in GCN related to nitrite and nitrate reduction in dry-fermented sausages and a similar effect was observed in Pastrima (Aksu et al., 2016). Similar findings were reported by GonzalesBarron et al. (2015) in dry-fermented sausages, although the presence in the formulation of polyphosphate, which produced a $\mathrm{pH}$ rise effect, was the main factor affecting the growth of acid sensitive microorganisms. In our formulations $\mathrm{C}$ and $\mathrm{R}$, the absence of differences in $\mathrm{pH}$ due to the similar LAB growth corroborated that the reduction of nitrate and nitrite levels did not affect GCN growth. Regarding the absence of Enterobacteriaceae, this indicated that the process was carried out with good hygiene practices, which are important to assure microbial safety. The inoculation of $D$. hansenii in RY sausages was confirmed by the yeasts and moulds counts during the whole process and presence of M13 RAPD PCR pattern of D. hansenii L1 (Fig. 2). The presence of $D$. hansenii L1 patterns in yeasts isolated from $\mathrm{C}$ and $\mathrm{R}$ sausages could be due to yeast cross contamination in the drying chamber. Nevertheless, the cell load (Figure 1) is very different between samples, leading to a scarce impact of these isolates in $\mathrm{R}$ and $\mathrm{C}$ sausages.

The main mechanisms involved in generation of volatile compounds with aroma impact on fermented sausages are from microbial origin, chemical oxidation (Flores \& Olivares, 2015) and thiamine degradation (Flores, 2018). Contrary to Hospital et al. (2015), total volatile compounds derived from amino acid degradation were not affected by the reduction of ingoing amounts of nitrate and nitrite. Besides, amino acid derived volatile compounds were in high abundance in sausages inoculated with $D$. hansenii strain L1 (Table 2) due to the yeast strain amino acid degradation capacity (Perea-Sanz et al., 2019a). On the other hand, our results indicated that $D$. hansenii might have consumed acetic acid and produced ethanol in contrast to what happened in C and R sausages (Table 2) (Cano-García et al., 2014). Esterase activity in dry-fermented sausages is carried out by Staphylococcus and Debaryomyces species (Sthanke, 1994; Flores, Dura, Marco \& Toldra, 2004). Accordingly, D. hansenii inoculation in RY sausages increased production of esters compounds (Flores et al., 2004). Morevoer, D. hansenii produced a strong decrease in lipid oxidation volatile compounds in agreement with the low TBARS values and the high antioxidant capacity of this yeast (Corral et al., 2015). Nitrate and nitrite reduction and D. hansenii did not affect volatile compounds derived from black pepper, which was used as spice in the formulations.

Generation of volatile compounds derived from amino acid degradation reactions in $\mathrm{C}$ and $\mathrm{R}$ sausages was not altered from 60 to $83 \mathrm{~d}$ (Table 2). However, RY sausages showed a decline in volatile compounds derived from amino acid degradation indicating a probable transformation of these into other compounds. The abundance of volatile compounds derived from lipid oxidation decreased in $\mathrm{C}$ and $\mathrm{R}$ sausages due to the further drying (Perea-Sanz et al., 2018). 
Concerning volatile compounds with direct impact on the sausage aroma profile (Table 3) at 60 $\mathrm{d}$ of ripening, the reduction of the ingoing amounts of nitrate and nitrite produced few aroma differences in contrast to the highest effect produced by $D$. hansenii, inoculation. The yeast prevented oxidation reactions and generation of aromas derived from lipid oxidation (Flores et al., 2004) which may produce unpleasant rancid odour notes in the sausages. In addition, it was responsible for the production of ester compounds which provided fruity and sweet odours to the sausages (Cano-García et al., 2014; Corral et al., 2015). The decrease on carbohydrate fermentation aromas caused by $D$. hansenii inoculation could be explained by the yeast consumption of acetic acid (Table 2). In general, D. hansenii inoculation decreased acid notes detrimental to sausage aroma (Flores, 1997). On the other hand, at $83 \mathrm{~d}$ of ripening the effect of nitrate and nitrite reduction between $\mathrm{C}$ and $\mathrm{R}$ on aroma profile is more clear (Figure 3C and D) (Perea-Sanz, Montero, Belloch \& Flores, 2019b) and D. hansenii yeast contributed to fruity notes (ester compounds) and prevented oxidation events. In addition, the relationship between nitrate/nitrite reduced fermented sausages (R) and the aroma compounds derived from carbohydrate fermentation (acetic acid, 2-butanone, 2,3-butanedione, 3-hydroxy-2-butanone, Figure 3D) may be explained by the GCN metabolic activity, although no differences in GCN growth were observed between nitrate/nitrite reduced and no-reduced sausages.

\section{Conclusions}

Nitrite and nitrate reduction in fermented sausages did not affect microbial growth but decreased lipid oxidation and generation of derived volatiles. Microorganisms metabolic activity caused differences in the aroma profile at the longest drying time. The nitrite prooxidant and antimicrobial effects were confirmed in C sausages. D. hansenii inoculation limited lipid oxidation and increased generation of volatile compounds derived from amino acid degradation and esterase activity. The antioxidant capacity of $D$. hansenii during the entire drying time prevented nitrite oxidation as demonstrated by the lowest residual nitrate levels in RY sausages. In summary, yeast inoculation counteracted the negative impact of nitrite and nitrate reduction on aroma due to its antioxidant capacity during drying time, aroma production and hindered nitrite oxidation.

\section{Acknowledgements}

Financial support from AGL2015-64673-R (AEI/FEDER, UE) and RTI2018-098074-B-I00 (MCIU/AEI/FEDER, UE), and the predoctoral scholarship (ACIF/2016/107, GVA, Spain) to L. Perea-Sanz are fully acknowledged. The authors are grateful to N. Fontana and Adriana A. Gordon for technical assistance. 


\section{References}

Aksu, M.I., Erdemir, E., Çakici, N. (2016). Changes in the physico-chemical and microbial quality during the production of pastırma cured with different levels of sodium nitrite. Korean Journal for Food Science of Animal Resources, 36, 617-625.

Alahakoon, A.U., Jayasena, D.D., Ramachandra, S., Jo, C. (2015). Alternatives to nitrite in processed meat: up to date. Trends Food Science Technology, 45, 37-39.

Aquilani, C., Sirtori, F., Flores, M., Bozzi, R., Lebert, B., Pugliese, C. (2018). Effect of natural antioxidants from grape seed and chestnut in combination with hydroxytyrosol, as sodium nitrite substitutes in Cinta Senese dry-fermented sausages. Meat Science, 145, 389-398.

Brannan, R.G., Connolly, B.J., Decker, E. (2001). Peroxynitrite: a potential initiator of lipid oxidation in food. Trends in Food Science \& Technology, 12, 164-173.

Burdok, G.A., (2002). Fenaroli's Handbook of Flavor Ingredients. (4th ed). Boca Raton. CRC Press Inc., Florida.

Cano-García, L., Flores, M., Belloch, C. (2013). Molecular characterization and aromatic potential of $D$. hansenii strains isolated from naturally fermented sausages. Food Chemistry, 151, 364-373.

Cano-García, L.; Belloch, C.; Flores, M. (2014). Impact of Debaryomyces hansenii strains inoculation on quality of slow dry-cured fermented sausages. Meat Science, 96, 14691477.

Christieans, S., Picgirard, L., Parafita, E., Lebert, A., Gregori, T. (2018). Impact of reducing nitrate/nitrite levels on the behavior of Salmonella typhimurium and Listeria monocytogenes in French dry fermented sausages. Meat science, 137, 160-167.

CODEX (2019) JOINT FAO/WHO FOOD STANDARDS PROGRAMME CODEX COMMITTEE ON FOOD ADDITIVES Fifty-first Session. Discussion paper on the use of nitrates (INS 251, 252) and nitrites (INS 249, 250). http://www.fao.org/fao-whocodexalimentarius/sh-

proxy/en/?lnk=1\&url=https $\% 253 \mathrm{~A} \% 252 \mathrm{~F} \% 252 \mathrm{Fworkspace}$.fao.org\% $\% 252 \mathrm{Fsites} \% 252 \mathrm{~F} \operatorname{codex} \% 2$ 52FMeetings\%252FCX-711-51\%252FWD\%252Ffa51_09e.pdf

Corral, S., Salvador, A., Belloch, C., Flores, M. (2015). Improvement the aroma of reduced fat and salt fermented sausages by Debaromyces hansenii inoculation. Food Control, $147,526-535$. 
De Mey, E., De Maere, H., Paelinck, H., Fraeye, I. (2015). Volatile N-nitrosamines in meat products: potential precursors, influence of processing, and mitigation strategies. Critical Reviews in Food Science and Nutrition, 57, 2909-2923.

European Food Safety Authority (EFSA), (2010). Statement on nitrites in meat products. EFSA Journal, 8, 1538.

Flores, J. (1997) Mediterranean vs northern European meat products. Processing technologies and main differences. Food Chemistry, 59, 505-510.

Flores, M., Dura, M.A., Marco, A., Toldrá, F. (2004). Effect of Debaryomyces spp. on aroma formation and sensory quality of dry-fermented sausages. Meat science, 68, 439-446.

Flores, M., Corral, S., Cano-García, L., Salvador, A., Belloch, C. (2015). Yeast strains as potential enhancers in dry fermented sausages. International Journal of Food Microbiology, $212,16-24$.

Flores, M., \& Olivares, A. (2015). Flavor. In F. Toldrá (Ed.), Handbook of Fermented Meat and Poultry (pp. 217-225). (2nd ed). John Wiley \& Sons, Ltd.

Flores, M., Moncunill, D., Montero, R., López-Díez, J. J., Belloch, C. (2017). Screening of Debaryomyces hansenii strains for flavour production under reduced concentration of nitrifying preservatives used in meat products. Journal of Agricultural and Food Chemistry, 65, 3900-3909.

Flores, M. (2018). Understanding the implications of current health trends on the aroma of wet and dry cured meat products. Meat Science, 144, 53-61.

Gonzales-Barron, U., Cadavez, V., Pereira, A.P., Gomes, A., Araújo, J.P., Saavedra, M.J., Estevinho, L., Butler, F., Pires, P., Dias, T. (2015). Relating physicochemical and microbiological safety indicators during processing of linguiça, a Portuguese traditional dryfermented sausage. Food Research International, 78, 50-61.

Gratacós-Cubarsí, M., Sárraga, C., Castellari, M., Valero, A., García-Regueiro, J.A., Arnau, J. (2013). Effect of pH 24h, curing salts and muscle types on oxidative stability, free amino acids profile and vitamin B2, B3 and B6 content of dry-cured ham. Food Chemistry, 141, 3207-3214.

Honikel, K.O. (2008). The use and control of nitrate and nitrite for the processing of meat products. Meat Science, $78,68-76$ 
Hospital, X.F., Hierro, E., Fernández, M. (2014). Effect of reducing nitrate and nitrite added to dry fermented sausages on the survival of Salmonella typhimurium. Food Research International, 62, 410-415.

Hospital, X.F., Carballo, J., Fernández, M., Arnau, J., Gratacós, M., Hierro, E. (2015). Technological implications of reducing nitrate and nitrite levels in dry-fermented sausages: typical microbiota, residual nitrate and nitrite and volatile profile. Food Control, 57, 275-581.

Hospital, X.F., Hierro, E., Stringer, S., Fernández, M. (2016). A study on the toxigenesis by Clostridium botulinum in nitrate and nitrite reduced dry fermented sausages. International Journal of Food Microbiology, 218, 66-70.

Majou, D., Christieans, S. (2018). Mechanisms of the bactericidal effects of nitrate and nitrite in cured meats. Meat Science, 145, 273-284

Merino, L. (2009). Development and validation of a method for determination of residual nitrite/nitrate in foodstuffs and water after zinc reduction. Food Analytical Methods, 2, 212-220.

Olivares, A., Navarro, J.L., Salvador, A., Flores, M. (2010). Sensory acceptability of slow fermented sausages based on fat content and ripening time. Meat Science, 86, 251-257.

Perea-Sanz, L., Montero, M., Belloch, C., \& Flores, M. (2018). Nitrate reduction in the fermentation process of salt reduced dry sausages: Impact on microbial safety, physicochemical parameters and aroma profile. International Journal of Food Microbiology, 282, 84 -91.

Perea-Sanz, L., Peris, D., Belloch, C., Flores, M. (2019a). Debaryomyces hansenii metabolism of sulfur amino acids as precursors of volatile sulfur compounds of interest in meat products. Journal of Agricultural and Food Chemistry, 67, 9335-9343.

Perea-Sanz, L., Montero, R., Belloch, C., Flores, M. (2019b). Microbial changes and aroma profile of nitrate reduced dry sausages during vacuum storage. Meat Science, 147, 100107.

Pollien, P., Ott, A., Montigon, F., Baumgartner, M., Muñoz-Box, R., Chaintreau, A. (1997). Hyphenated headspace-gas chromatography-sniffing technique: screening of impact odorants and quantitative aromagram comparisons. Journal of Agricultural and Food Chemistry, 45, 2630-2637.

Regulation (EC) No 1333/2008 of the European Parliament and of the Council of 16 December 2008 on food additives. ANNEX II (Part E). (pp. 170 and 182). 
Sánchez Mainar, M., Leroy, F. (2015). Process-driven bacterial community dynamics are key to cured meat colour formation by coagulase-negative staphylococci via nitrate reductase or nitric oxide synthase activities. International Journal of Food Microbiology, 212, $60-66$.

Sindelar, J.J., Milkowski, A.L. (2011). Sodium nitrite in processed meat and poultry meats: a review of curing and examining the risk/benefit of its use. In: American Meat Science Association (AMSA). White Paper Series. 3.

Sthanke, L.H. (1994). Aroma components from dried sausages fermented with Staphylococcus xylosus. Meat Science, 38, 39-53.

Van Den Dool, H., Kratz, P.D. (1963). A generalization of the retention index system including linear temperature programmed gas-liquid partition chromatography. Journal of Chromatography, 2, 463-471.

Zanardi, E., Ghidini, S., Battaglia, A., Chizzolini, R. (2004). Lipolysis and lipid oxidation in fermented sausages depending on different processing conditions and different antioxidants. Meat Science, 66, 415-423. 